Zagazig J. Agric. Res., Vol. 43 No. (3) 2016

http:/www.journals.zu.edu.eg/journalDisplay.aspx?Journalld=1\&queryType=Master

\title{
HETEROSIS AND FACTOR ANALYSIS FOR SOME IMPORTANT TRAITS IN NEW MAIZE HYBRIDS
}

\author{
Youstina S. Sedhom*, M.M.A. Ali, H.A. Awaad and H.A. Rabie \\ Agron. Dept., Fac. Agric., Zagazig Univ., Egypt
}

\begin{abstract}
The present study was performed to determine the extent of standard heterosis in forty five crosses resulting from a $10 \times 10$ diallel analysis system. The obtained crosses along with two checks (S.C. 10 and S.C. $30 \mathrm{k} 8$ ) were evaluated in two different planting dates i.e., May, $15^{\text {th }}$ and June $15^{\text {th }}$. Standard heterosis were computed for days to $50 \%$ tasseling, days to $50 \%$ silking, plant height, ear height, chlorophyll content, grain yield / plant, protein (\%) and oil (\%) in each planting date as well as combined analyses. Results indicated that the single cross $\mathrm{P}_{5} \times \mathrm{P}_{6}$ expressed the highest desirable heterotic values for days to $50 \%$ tasseling and chlorophyll content relative to both checks. The hybrid $\mathrm{P}_{4} \times \mathrm{P}_{5}$ gave the best standard heterotic effects for days to $50 \%$ silking relative to both checks. The best standard heterosis for plant height and ear height relative to both checks was obtained for the cross $\mathrm{P}_{1} \times \mathrm{P}_{8}$ in the second planting date and combined data. For grain yield/plant, the most desirable standard heterotic effects were obtained for the cross $\mathrm{P}_{6} \times \mathrm{P}_{8}$ relative to S.C. 10 and S.C. $30 \mathrm{k} 8$, recording $20.10 \%$ and $17.72 \%$ in the combined analyses, respectively. The best standard heterosis effects for protein (\%) were detected in the cross $\mathrm{P}_{4} \times \mathrm{P}_{7}$ relative to both checks in the second planting date and the combined analyses. For oil (\%), the best heterosis values relative to both checks were detected for the cross $\mathrm{P}_{1} \times \mathrm{P}_{2}$ in the combined analyses. The correlation coefficient values between grain yield/plant and each of chlorophyll content, ear length, ear diameter, number of rows/ear, number of grains/row, 100 grain weight and shelling (\%) were positive and highly significant. The factor analysis technique divided the studied variables into four main factors accounted for $77.83 \%$ of the total variance. The first factor included four variables i.e., ear length, number of rows/ear, number of grains/row and shelling (\%) and accounted for $21.15 \%$. While, the second factor consisted of two variables i.e., plant and ear heights and accounted for $19.73 \%$ of the total variance. The third factor included two variables namely, days to $50 \%$ tasseling and silking and accounted for $19.00 \%$ of total variance. Three variables were loaded in the fourth factor i.e., chlorophyll content, ear diameter and 100 grain weight and accounted for $17.95 \%$ of the total variance of the dependence structure.
\end{abstract}

Key words: Maize, standard heterosis, correlation, factor analysis.

\section{INTRODUCTION}

Maize (Zea mays, L.) is one of the most important cereal crops in Egypt and the world. It ranks the third among the world cereal crops, surpassed only by wheat and rice. In 2013 the area allotted to this crop in Egypt was 1.724 million faddans and total production was 5.788.000 million tons of grains with an average yield of $23.98 \mathrm{ardab} /$ faddan. However, the total production is far less than that required for local

\footnotetext{
* Corresponding author. Tel.: +201281230452

E-mail address: ystn_sedhom@yahoo.com
}

consumption. Recently, efforts have been made to increase food and agricultural production, mainly through developing new hybrids characterized by high yielding potentiality and better quality to fill the gap between maize production and consumption. This depends mainly upon the exploitation of heterosis in maize breeding program.

Heterosis is the phenomenon in which the cross of two parents produces hybrid that is superior in growth, size, yield, or vigor of the $F_{1}$ 
over the better parent. It has been extensively studied in maize because of (i) its large expression for grain yield (100 - 200\%), (ii) its intensive exploitation in hybrid breeding of maize, and (iii) the favourable biological prerequisites such as large multiplication coefficient and easy of both self and controlled cross- fertilization (Ram et al., 2015). The magnitude of heterosis provides formation on extent of genetic diversity of parents in developing superior $\mathrm{F}_{1}$ s so as to exploit hybrid vigour and has direct bearing on the breeding methodology to be adapted for varietal improvement (Rajesh et al., 2014). Several investigators calculated heterosis in maize over mid-parent (relative heterosis), over betterparent (heterobeltiosis) and over check (standard heterosis). Among those are (Ali et al., 2014; Abrha, 2014; Ram et al., 2015). In this concern, significant standard heterosis for grain yield and its contributing traits were detected and revealed that it can be used as a tool for high productivity in maize (Zaid et al., 2014; Reddy et al., 2015). Abrha (2014) concluded that the presence of substantial heterotic potential could be exploited in maize breeding program for the developing of desirable cross combinations and synthetic varieties through crossing and/ or recombination of inbred lines with desirable traits of interest.

Also, maize breeders are interested in studying the nature of relationship between maize grain yield and its contributing traits which help the breeders to select the best genotypes based on yield and related characters. Therefore, applying simple correlation and factor analysis are very helpful since factor analysis is a type of multivariate analysis that can be used to reduce a large number of correlated variables to a smaller number of main factors (Beshay, 2010; Beiragi et al. 2012; Khodarahmpour, 2013). Factor analysis is used by plant breeders because it has the potential of increasing the comprehension of causal relationship of variables and can help to determine the nature and sequence of traits to be selected in breeding program. Filipovic et al. (2014) determined the interrelationships of yield and yield components of 15 commercial maize hybrids using factor analysis and extracted two main factors responsible for $60.51 \%$ of total variability. The first factor accounted for $47.71 \%$, while the second factor accounted for $12.8 \%$ of the total variance in the dependence structure. Sayedzavar et al. (2015) studied factor analysis and found that two factors justified $78 \%$ of total variance. The first factor $(50.8 \%)$ had the great coefficient on 300-grain weight, plant height, length of ear, ear leaf area, ear diameter and cob diameter. The second factor $(27.2 \%)$ had the great coefficient on plant dry weight, number of grains per row, number of leaves per plant, number of rows per ear and grain yield. Therefore, the objectives of this investigation were to: 1- Identify superior hybrids to improve the yielding ability in maize breeding program. 2- Studying correlation and factor analysis for several variables related to maize grain yield.

\section{MATERIALS AND METHODS}

Ten diverse parental inbred lines of white maize (Zea mays L.) were used in this study i.e. $\mathrm{P}_{1}, \mathrm{P}_{2}, \mathrm{P}_{3}, \mathrm{P}_{4}, \mathrm{P}_{5}, \mathrm{P}_{6}, \mathrm{P}_{7}, \mathrm{P}_{8}, \mathrm{P}_{9}$ and $\mathrm{P}_{10}$. These parental inbred lines were isolated from different genetic resources and were at $\mathrm{S}_{8}$ stage of inbreeding. All these parental inbreds were developed at the Department of Agronomy, Faculty of Agriculture, Benha University, by Prof. Dr. S.A. Sedhom and represented a wide range of variability for yield and most of its attributes. The names, origin and characteristics of these inbred lines are presented in Table 1. A half diallel set of crosses was carried out in 2013 season. The ten inbred lines were split planted on May $15^{\text {th }}, 25^{\text {th }}$ and June $5^{\text {th }}$ to avoid differences in flowering time and to secure enough hybrid seeds. In 2014 summer season, the resultant 45 crosses along with two checks (S.C. 10 and S.C. $30 \mathrm{k} 8$ ) were planted in a randomized complete block design with three replications on two planting dates, i.e., $15^{\text {th }}$ May and $15^{\text {th }}$ June at the Agricultural Research and Experimental Station of the Fac. Agric., Benha University. On each planting date, experimental plot consisted of one ridge of three $m$ length and $70 \mathrm{~cm}$ width. Hills were spaced by $25 \mathrm{~cm}$ with three seeds per hill on one side of the ridge. The seedlings were later thinned to one plant per hill. The cultural practices were followed properly for ordinary maize field in the area. 
Table 1. The name, origin and characteristics of the studied ten parental inbred lines

\begin{tabular}{|c|c|c|c|c|c|c|}
\hline $\begin{array}{l}\text { Parent } \\
\text { code }\end{array}$ & $\begin{array}{c}\text { Parent } \\
\text { name }\end{array}$ & Origin & $\begin{array}{l}\text { Days to } 50 \% \\
\text { silking }\end{array}$ & $\begin{array}{l}\text { Plant height } \\
\text { (cm) }\end{array}$ & $\begin{array}{c}\text { No. of rows } \\
\text { ear }^{-1}\end{array}$ & $\begin{array}{c}\text { Grain yield }(\mathrm{g}) \\
\text { plant }^{-1}\end{array}$ \\
\hline $\mathrm{P} 1$ & M2 & Cairo 1 & $66.80 \pm 0.37$ & $251.20 \pm 0.80$ & $12.40 \pm 0.40$ & $35.60 \pm 1.96$ \\
\hline $\mathrm{P} 2$ & M3 & Cairo 1 & $68.40 \pm 0.51$ & $260.40 \pm 0.51$ & $12.40 \pm 0.97$ & $32.40 \pm 1.12$ \\
\hline P3 & M5 & Cairo 1 & $57.20 \pm 0.58$ & $234.00 \pm 1.18$ & $14.40 \pm 0.40$ & $40.80 \pm 1.02$ \\
\hline P4 & M10 & Giza 2 & $60.00 \pm 0.71$ & $195.00 \pm 2.54$ & $12.00 \pm 0.63$ & $64.20 \pm 0.97$ \\
\hline P5 & M12 & Giza 2 & $57.50 \pm 0.37$ & $140.00 \pm 1.70$ & $14.00 \pm 0.63$ & $89.60 \pm 1.63$ \\
\hline P6 & M14 & Giza 2 & $64.80 \pm 0.37$ & $158.20 \pm 1.85$ & $12.40 \pm 0.40$ & $62.40 \pm 1.36$ \\
\hline P7 & M19 & Giza 2 & $62.80 \pm 0.37$ & $162.00 \pm 2.02$ & $12.00 \pm 0.63$ & $40.80 \pm 1.32$ \\
\hline P8 & M20 & Pioneer 514 & $64.20 \pm 0.58$ & $187.20 \pm 1.16$ & $13.60 \pm 0.74$ & $78.60 \pm 2.04$ \\
\hline P9 & M21 & Pioneer 514 & $61.80 \pm 0.58$ & $214.00 \pm 1.87$ & $12.00 \pm 0.63$ & $52.20 \pm 1.24$ \\
\hline P10 & M24 & Pioneer 514 & $68.20 \pm 0.37$ & $176.00 \pm 1.87$ & $12.40 \pm 0.40$ & $45.60 \pm 1.12$ \\
\hline
\end{tabular}

\section{Data Recorded}

\section{Earliness traits}

In each experimental plot, days to $50 \%$ tasseling and days to $50 \%$ silking were estimated.

\section{Morphophysiological traits}

Plant height $(\mathrm{cm})$, ear height $(\mathrm{cm})$ and chlorophyll content (SPAD value) of ear leaf were measured from 10 random plants in each plot in each replicate.

\section{Yield and its attributes}

Random sample of 10 guarded plants in each plot was taken to evaluate ear length $(\mathrm{cm})$ ear diameter $(\mathrm{cm})$, No. of grains/row, No. of rows/ ear, shelling (\%), 100-grain weight, and grain yield/plant which was adjusted for $15.5 \%$ moisture.

\section{Quality traits}

Protein (\%) and oil (\%) were determined according to AOAC (1990).

\section{Statistical Analysis}

The obtained data were statistically analyzed for each planting date and the combined analysis was performed whenever homogeneity of variance was detected (Gomez and Gomez, 1984). Standard heterosis was calculated for days to $50 \%$ tasseling, days to $50 \%$ silking, chlorophyll content, grain yield / plant, protein $(\%)$ and oil (\%) as follows:

Standard heterosis $=\left[\frac{F_{1}-\text { check variety }}{\text { check variety }} \times 100\right]$

Appropriate LSD values were computed according to the following formulae to test the significance of heterotic effects.

LSD for heterosis relative to check variety $=$

$$
\mathrm{tx} \sqrt{\frac{2 \mathrm{MSe}}{\mathrm{r}}}
$$

Where:

$\mathrm{t}$ : is the tabulated $\mathrm{t}$ value at a stated level of probability for the experimental error degree of freedom.

$\mathrm{r}$ : is the number of replications.

Simple correlation coefficient was estimated according to Snedecor and Cochran (1967). Correlation coefficients were used to calculate factor analysis according to Cattell (1965).

\section{RESULTS AND DISCUSSION}

\section{Standard Heterosis}

Superiority of studied crosses over both checks (S.C. 10 and S.C. $30 \mathrm{k} 8$ ) in the first and 
second planting dates as well as combined analysis were calculated for different traits as follows:

\section{Earliness traits}

Standard heterosis values for days to $50 \%$ tasseling and days to $50 \%$ silking in each planting date as well as combined data are presented in Tables 2 and 3. For days to 50\% tasseling, sixteen, twenty six and twenty six crosses exhibited significant and negative standard heterotic values relative to S.C. 10; eleven, twenty three and twenty three hybrids relative to S.C. $30 \mathrm{k} 8$ in the first, second planting dates and combined analysis, respectively (Table 2). However, the single cross $\mathrm{P}_{5} \mathrm{x}_{6}$ expressed the most desirable heterotic effect for this trait recoding $-11.54,-11.76$ and $-11.65 \%$ relative to S.C. 10 and $-10.56,-11.24$ and -10.89 relative to S.C. $30 \mathrm{k} 8$ in the first, second planting dates and combined data, respectively.

For days to $50 \%$ silking, seven, thirty six and thirty one crosses expressed significant and negative standard heterotic values relative to S.C. 10 in the first, second planting dates and combined analysis, respectively. While, six, fourteen and sixteen hybrids exhibited significant and negative heterotic effects relative to S.C. $30 \mathrm{k} 8$ in the same order (Table 3). However, the hybrid $\mathrm{P}_{4} \times \mathrm{P}_{5}$ gave the best standard heterotic effects being -10.94, -17.53 and $-14.25 \%$ relative to S.C. 10 and -10.47 , 12.57 and $-11.50 \%$ relative to S.C. $30 \mathrm{k} 8$ in the first and second planting dates as well as combined data, respectively.

Earliness if found in corn crosses is favourbale for escaping destructive injuries caused by Sesamia cretica ledi chilo simplex and Pyrausta nubilalis. Similar results were obtained by Abd-Elaziz (2014), Abrha (2014), Al- Falahy (2015) and Reddy et al. (2015) who found significant and negative standard heterosis for days to $50 \%$ tasseling and days to $50 \%$ silking.

\section{Morphophysiological traits}

Standard heterosis for plant height, ear height and chlorophyll content relative to both checks in the first and second planting date as well as combined analysis are presented in Tables 4, 5 and 6.
Regarding plant height, standard heterosis relative to S.C. 10 ranged from -22.59 to 3.67 ; -27.82 to -2.59 and -21.42 to 0.51 in the first, second planning dates as well as combined analysis, respectively. The respective heterotic values for the check S.C. $30 \mathrm{k} 8$ ranged from -18 . 67 to $8.92 ;-26.32$ to -0.57 and -18.65 to $4.06 \%$. However, the single cross $\mathrm{P}_{5} \times \mathrm{p}_{7}$ exhibited the best heterotic values in the first planting date, while the cross $\mathrm{P}_{1} \times \mathrm{P}_{8}$ expressed the most desirable standard heterosis for plant height in the second planting date as well as combined analysis relative to both checks.

For ear height, heterotic effects relative to S.C. 10 ranged from -19.87 to $8.04 ;-26.83$ to -0.44 and -21.25 to $1.67 \%$ in the first, second planting dates as well as combined data, respectively. The heterotic values relative to S.C. $30 \mathrm{k} 8$ ranged from -13.91 to $16.07 ;-20.29$ to 8.45 and -14.80 to $9.99 \%$ for the respective cases. However, the best standard heterosis effects were detected for the cross $\mathrm{P}_{5} \times \mathrm{P}_{7}$ in the first plant date relative to S.C. $10(-19.87 \%)$ and S.C. $30 \mathrm{k} 8(-13.91 \%)$. Meanwhile, the cross $\mathrm{P}_{1} \mathrm{x}$ $\mathrm{P}_{8}$ gave the best heterotic effect in the second planting date $(-26.83$ and $-20.29 \%)$ as well as combined analysis $(-21.25$ and $-14.80 \%)$ relative to S.C. 10 and S.C. $30 \mathrm{k} 8$, respectively. These results of plant and ear heights are in agreement with those reported by Abd-Elaziz (2014), Abdel-Moneam et al. (2014), Abrha (2014) and Zaid et al. (2014).

For Chlorohphyll content, eight, nine and twelve crosses exhibited significant and positive heterotic effects relative to S.C. 10 in the first, second planting dates and combined data, respectively. Significant and positive standard heterosis relative to SC $30 \mathrm{k} 8$ were detected for six, five and eight crosses in the same manner. However, the most desirable heterotic effects for chlorophyll content were detected for the cross $\mathrm{P}_{5} \times \mathrm{P}_{6}$ recording $14.36 ; 27.38$ and $20.61 \%$ relative to S.C. 10 and $10.56,21.20$ and $15.70 \%$ relative to $\mathrm{SC} 30 \mathrm{k} 8$, in the first, second planting date and combined data, respectively (Table 6). Moreover, the best heterotic effect for this trait was recorded by the cross $\mathrm{P}_{5} \times \mathrm{P}_{6}(20.61$ and $15.70 \%)$ followed by the cross $\mathrm{P}_{6} \times \mathrm{P}_{8}(16.40$ and $11.66 \%)$ then the cross $\mathrm{P}_{6} \times \mathrm{P}_{10}(16.24$ and $11.51 \%$ ) relative to S.C. 10 and SC $30 \mathrm{k} 8$ in the combined data. These results are in accordance with Ulaganathan and Ibrahim (2014). 
Table 2. Heterosis for days to $50 \%$ tasseling relative to S.C. 10 and S.C. $30 \mathrm{K8}$ in two planting dates as well as combined data

\begin{tabular}{|c|c|c|c|c|c|c|}
\hline \multirow{3}{*}{$\begin{array}{l}\text { Trait } \\
\text { Cross }\end{array}$} & \multicolumn{6}{|c|}{ Days to $50 \%$ tasseling } \\
\hline & \multicolumn{3}{|c|}{ Heterosis (\%) relative to S.C. 10} & \multicolumn{3}{|c|}{ Heterosis (\%) relative to S.C. 30 K 8} \\
\hline & D1 & D2 & Com. & D1 & D2 & Com. \\
\hline $\mathbf{P 1} \times \mathbf{P 2}$ & $-8.24 *$ & $-8.82 * *$ & $-8.52 * *$ & $-7.22 *$ & $-8.28 * *$ & $-7.74 * *$ \\
\hline $\mathbf{P 1} \times \mathbf{P 3}$ & -6.04 & $-8.24 * *$ & $-7.10 * *$ & -5.00 & $-7.69 * *$ & $-6.30 * *$ \\
\hline $\mathbf{P 1} \times \mathbf{P 4}$ & -6.04 & $-5.88 *$ & $-5.97 * *$ & -5.00 & $-5.33 *$ & $-5.16^{*}$ \\
\hline P1 $\times$ P5 & -3.30 & $-5.29 *$ & -4.26 & -2.22 & -4.73 & -3.44 \\
\hline $\mathbf{P 1} \times \mathbf{P 6}$ & -1.10 & $-6.47^{*}$ & -3.69 & 0.00 & $-5.92 *$ & -2.87 \\
\hline $\mathbf{P 1} \times \mathbf{P 7}$ & -4.40 & -4.71 & $-4.55^{*}$ & -3.33 & -4.14 & -3.72 \\
\hline P1 $\times$ P8 & -2.75 & 0.00 & -1.42 & -1.67 & 0.59 & -0.57 \\
\hline $\mathbf{P 1} \times \mathbf{P 9}$ & -3.30 & $-11.18 * *$ & $-7.10 * *$ & -2.22 & $-10.65 * *$ & $-6.30 * *$ \\
\hline $\mathbf{P} 1 \times \mathbf{P} 10$ & 6.04 & -2.35 & 1.99 & $7.22 *$ & -1.78 & 2.87 \\
\hline $\mathbf{P} 2 \times \mathbf{P 3}$ & $11.54 * *$ & $-8.24 * *$ & 1.99 & $12.78 * *$ & $-7.69 * *$ & 2.87 \\
\hline $\mathbf{P 2} \times \mathbf{P 4}$ & -6.04 & $-10.59 * *$ & $-8.24 * *$ & -5.00 & $-10.06 * *$ & $-7.45 * *$ \\
\hline $\mathbf{P 2} \times \mathbf{P 5}$ & -4.40 & -3.53 & -3.98 & -3.33 & -2.96 & -3.15 \\
\hline $\mathbf{P 2} \times \mathbf{P 6}$ & 3.30 & $-7.06 * *$ & -1.70 & 4.44 & $-6.51 *$ & -0.86 \\
\hline $\mathbf{P 2} \times \mathbf{P 7}$ & -2.20 & $-5.88^{*}$ & -3.98 & -1.11 & $-5.33 *$ & -3.15 \\
\hline $\mathbf{P 2} \times \mathbf{P 8}$ & $-7.69 *$ & $-8.24 * *$ & $-7.95 * *$ & -6.67 & $-7.69 * *$ & $-7.16 * *$ \\
\hline $\mathbf{P 2} \times \mathbf{P 9}$ & -3.85 & $-11.76^{* *}$ & $-7.67 * *$ & -2.78 & $-11.24 * *$ & $-6.88 * *$ \\
\hline $\mathbf{P} 2 \times \mathbf{P} 10$ & $-8.24 *$ & $-8.24 * *$ & $-8.24 * *$ & $-7.22 *$ & $-7.69 * *$ & $-7.45 * *$ \\
\hline P3 $\times$ P4 & $-7.14^{*}$ & $-9.41 * *$ & $-8.24 * *$ & -6.11 & $-8.88 * *$ & $-7.45 * *$ \\
\hline P3 $\times$ P5 & -2.75 & -1.76 & -2.27 & -1.67 & -1.18 & -1.43 \\
\hline $\mathbf{P 3} \times \mathbf{P 6}$ & -6.59 & $-6.47 *$ & $-6.53 * *$ & -5.56 & $-5.92 *$ & $-5.73 *$ \\
\hline $\mathbf{P 3} \times \mathbf{P 7}$ & $-8.79 *$ & $-5.29 *$ & $-7.10 * *$ & $-7.78 *$ & -4.73 & $-6.30 * *$ \\
\hline $\mathbf{P 3} \times \mathbf{P 8}$ & $-9.89 * *$ & $-5.88 *$ & $-7.95 * *$ & $-8.89 *$ & $-5.33 *$ & $-7.16 * *$ \\
\hline $\mathbf{P 3} \times \mathbf{P 9}$ & -1.65 & $-10.00 * *$ & $-5.68 *$ & -0.56 & $-9.47 * *$ & $-4.87^{*}$ \\
\hline $\mathbf{P 3} \times \mathbf{P 1 0}$ & $-7.14^{*}$ & $-7.06 * *$ & $-7.10 * *$ & -6.11 & $-6.51 *$ & $-6.30 * *$ \\
\hline P4 $\times$ P5 & $-11.54 * *$ & $-11.18 * *$ & $-11.36 * *$ & $-10.56^{* *}$ & $-10.65 * *$ & $-10.60 * *$ \\
\hline P4 $\times$ P6 & $-9.34 * *$ & $-10.00 * *$ & $-9.66 * *$ & $-8.33 *$ & $-9.47 * *$ & $-8.88 * *$ \\
\hline $\mathbf{P 4} \times \mathbf{P 7}$ & $-9.89 * *$ & $-5.29 *$ & $-7.67 * *$ & $-8.89 *$ & -4.73 & $-6.88 * *$ \\
\hline $\mathrm{P} 4 \times \mathrm{P8}$ & $-10.99 * *$ & $-6.47^{*}$ & $-8.81 * *$ & $-10.00 * *$ & $-5.92 *$ & $-8.02 * *$ \\
\hline P4 $\times$ P9 & $-9.89 * *$ & $-11.76^{* *}$ & $-10.80 * *$ & $-8.89 *$ & $-11.24 * *$ & $-10.03 * *$ \\
\hline $\mathbf{P 4} \times \mathbf{P 1 0}$ & -6.59 & -1.76 & -4.26 & -5.56 & -1.18 & -3.44 \\
\hline $\mathbf{P 5} \times \mathbf{P 6}$ & $-11.54 * *$ & $-11.76^{* *}$ & $-11.65 * *$ & $-10.56^{* *}$ & $-11.24 * *$ & $-10.89 * *$ \\
\hline P5 $\times$ P7 & $-8.24 *$ & -3.53 & $-5.97 * *$ & $-7.22 *$ & -2.96 & $-5.16^{*}$ \\
\hline $\mathbf{P 5} \times \mathbf{P 8}$ & -4.95 & -0.59 & -2.84 & -3.89 & 0.00 & -2.01 \\
\hline P5 $\times$ P9 & -2.20 & $-7.65 * *$ & $-4.83 *$ & -1.11 & $-7.10 * *$ & -4.01 \\
\hline P5 $\times$ P10 & -3.30 & $10.59 * *$ & 3.41 & -2.22 & $11.24 * *$ & 4.30 \\
\hline $\mathbf{P 6} \times \mathbf{P 7}$ & -5.49 & -3.53 & $-4.55^{*}$ & -4.44 & -2.96 & -3.72 \\
\hline $\mathrm{P6} \times \mathrm{P8}$ & $-7.14 *$ & -3.53 & $-5.40 *$ & -6.11 & -2.96 & $-4.58 *$ \\
\hline P6 $\times$ P9 & -3.30 & 0.59 & -1.42 & -2.22 & 1.18 & -0.57 \\
\hline $\mathrm{P} 6 \times \mathbf{P} 10$ & -3.30 & $5.29 *$ & 0.85 & -2.22 & $5.92 *$ & 1.72 \\
\hline $\mathbf{P 7} \times \mathbf{P 8}$ & -6.59 & -1.76 & -4.26 & -5.56 & -1.18 & -3.44 \\
\hline P7 $\times$ P9 & $-7.69 *$ & -4.12 & $-5.97 * *$ & -6.67 & -3.55 & $-5.16^{*}$ \\
\hline $\mathbf{P} 7 \times \mathbf{P} 10$ & -2.75 & $8.82 * *$ & 2.84 & -1.67 & $9.47 * *$ & 3.72 \\
\hline $\mathbf{P 8} \times \mathbf{P 9}$ & -6.04 & -1.18 & -3.69 & -5.00 & -0.59 & -2.87 \\
\hline $\mathbf{P 8} \times \mathbf{P 1 0}$ & $10.44 * *$ & $10.00 * *$ & $10.23 * *$ & $11.67 * *$ & $10.65 * *$ & $11.17 * *$ \\
\hline $\mathbf{P 9} \times \mathbf{P 1 0}$ & 1.65 & $8.82 * *$ & $5.11 *$ & 2.78 & $9.47 * *$ & $6.02 * *$ \\
\hline
\end{tabular}

D1, D2 and com. refer to first, second planting dates and combined data, respectively.

$*$ and ** significant at 0.05 and 0.01 levels of probability, respectively. 
Table 3. Heterosis for days to $50 \%$ silking relative to S.C. 10 and S.C. $30 \mathrm{K8}$ in two planting dates as well as combined data

\begin{tabular}{|c|c|c|c|c|c|c|}
\hline \multirow{3}{*}{$\begin{array}{l}\text { Trait } \\
\text { Cross } \\
\end{array}$} & \multicolumn{6}{|c|}{ Days to $50 \%$ silking } \\
\hline & \multicolumn{3}{|c|}{ Heterosis (\%) relative to S.C. 10} & \multicolumn{3}{|c|}{ Heterosis (\%) relative to S.C. $30 \mathrm{~K} 8$} \\
\hline & D1 & D2 & Com. & D1 & D2 & Com. \\
\hline$\overline{\mathbf{P 1} \times \mathbf{P 2}}$ & -3.13 & $-7.73 *$ & $-5.44 *$ & -2.62 & -2.19 & -2.41 \\
\hline $\mathbf{P 1} \times \mathbf{P 3}$ & -0.52 & $-11.34 * *$ & $-5.96 * *$ & 0.00 & -6.01 & -2.94 \\
\hline P1 $\times$ P4 & -4.69 & $-12.37 * *$ & $-8.55^{* *}$ & -4.19 & $-7.10^{*}$ & $-5.61 *$ \\
\hline P1 $\times$ P5 & 1.04 & $-7.22 *$ & -3.11 & 1.57 & -1.64 & 0.00 \\
\hline$P 1 \times P 6$ & 1.56 & $-10.82 * *$ & $-4.66^{*}$ & 2.09 & -5.46 & -1.60 \\
\hline $\mathbf{P 1} \times \mathbf{P 7}$ & -2.60 & $-10.31 * *$ & $-6.48 * *$ & -2.09 & -4.92 & -3.48 \\
\hline $\mathbf{P 1} \times \mathbf{P 8}$ & 1.04 & 0.00 & 0.52 & 1.57 & 6.01 & 3.74 \\
\hline $\mathbf{P 1} \times \mathbf{P 9}$ & 0.52 & $-17.01 * *$ & $-8.29 * *$ & 1.05 & $-12.02 * *$ & $-5.35^{*}$ \\
\hline $\mathbf{P} 1 \times \mathbf{P} 10$ & 5.73 & $-8.76^{* *}$ & -1.55 & $6.28^{*}$ & -3.28 & 1.60 \\
\hline $\mathbf{P 2} \times \mathbf{P 3}$ & $9.90^{* *}$ & $-12.37 * *$ & -1.30 & $10.47 * *$ & $-7.10^{*}$ & 1.87 \\
\hline P2 $\times \mathbf{P 4}$ & -4.69 & $-16.49 * *$ & $-10.62 * *$ & -4.19 & $-11.48 * *$ & $-7.75^{* *}$ \\
\hline $\mathbf{P 2} \times \mathbf{P 5}$ & -1.56 & $-9.79 * *$ & $-5.70 * *$ & -1.05 & -4.37 & -2.67 \\
\hline $\mathbf{P 2} \times \mathbf{P 6}$ & 2.08 & $-12.89 * *$ & $-5.44 *$ & 2.62 & $-7.65^{*}$ & -2.41 \\
\hline $\mathbf{P} 2 \times \mathbf{P 7}$ & 2.60 & $-10.82 * *$ & -4.15 & 3.14 & -5.46 & -1.07 \\
\hline $\mathbf{P} 2 \times \mathbf{P 8}$ & -4.69 & $-12.37 * *$ & $-8.55^{* *}$ & -4.19 & $-7.10 *$ & $-5.61 *$ \\
\hline $\mathbf{P 2} \times \mathbf{P 9}$ & -3.13 & $-7.22 *$ & $-5.18^{*}$ & -2.62 & -1.64 & -2.14 \\
\hline $\mathbf{P} 2 \times \mathbf{P} 10$ & -4.69 & $-13.92 * *$ & $-9.33 * *$ & -4.19 & $-8.74 * *$ & $-6.42 * *$ \\
\hline P3 $\times$ P4 & -3.65 & $-16.49 * *$ & $-10.10^{* *}$ & -3.14 & $-11.48 * *$ & $-7.22 * *$ \\
\hline P3 $\times$ P5 & 0.00 & -4.12 & -2.07 & 0.52 & 1.64 & 1.07 \\
\hline $\mathbf{P 3} \times \mathbf{P 6}$ & -3.13 & $-10.31 * *$ & $-6.74 * *$ & -2.62 & -4.92 & -3.74 \\
\hline $\mathbf{P 3} \times \mathbf{P 7}$ & -4.69 & $-7.73 *$ & $-6.22 * *$ & -4.19 & -2.19 & -3.21 \\
\hline $\mathbf{P 3} \times \mathbf{P 8}$ & $-6.25^{*}$ & $-9.28 * *$ & $-7.77 * *$ & -5.76 & -3.83 & $-4.81 *$ \\
\hline $\mathbf{P 3} \times \mathbf{P 9}$ & 1.04 & $-14.95 * *$ & $-6.99 * *$ & 1.57 & $-9.84 * *$ & -4.01 \\
\hline $\mathbf{P 3} \times \mathbf{P 1 0}$ & -4.69 & $-11.34 * *$ & $-8.03 * *$ & -4.19 & -6.01 & $-5.08 *$ \\
\hline P4 × P5 & $-10.94 * *$ & $-17.53 * *$ & $-14.25^{* *}$ & $-10.47 * *$ & $-12.57 * *$ & $-11.50 * *$ \\
\hline P4 × P6 & -5.21 & $-17.01 * *$ & $-11.14^{* *}$ & -4.71 & $-12.02 * *$ & $-8.29 * *$ \\
\hline P4 $\times \mathbf{P 7}$ & $-7.29 *$ & $-11.34 * *$ & $-9.33 * *$ & $-6.81 *$ & -6.01 & $-6.42 * *$ \\
\hline P4 $\times$ P8 & $-8.33 * *$ & $-10.82 * *$ & $-9.59^{* *}$ & $-7.85^{*}$ & -5.46 & $-6.68 * *$ \\
\hline P4 × P9 & $-7.29 *$ & $-17.01 * *$ & $-12.18^{* *}$ & $-6.81 *$ & $-12.02 * *$ & $-9.36^{* *}$ \\
\hline $\mathbf{P 4} \times \mathbf{P 1 0}$ & -4.69 & $-8.25 * *$ & $-6.48 * *$ & -4.19 & -2.73 & -3.48 \\
\hline P5 $\times$ P6 & $-9.90 * *$ & $-17.01 * *$ & $-13.47 * *$ & $-9.42 * *$ & $-12.02 * *$ & $-10.70^{* *}$ \\
\hline P5 $\times \mathbf{P 7}$ & $-7.29 *$ & $-9.28 * *$ & $-8.29 * *$ & $-6.81 *$ & -3.83 & $-5.35^{*}$ \\
\hline $\mathbf{P 5} \times \mathbf{P 8}$ & -3.65 & -4.64 & -4.15 & -3.14 & 1.09 & -1.07 \\
\hline P5 $\times$ P9 & -0.52 & $-11.86^{* *}$ & $-6.22 * *$ & 0.00 & $-6.56^{*}$ & -3.21 \\
\hline P5 $\times$ P10 & 0.00 & 3.61 & 1.81 & 0.52 & $9.84 * *$ & $5.08^{*}$ \\
\hline $\mathbf{P 6} \times \mathbf{P 7}$ & -3.13 & $-10.82 * *$ & $-6.99 * *$ & -2.62 & -5.46 & -4.01 \\
\hline $\mathbf{P 6} \times \mathbf{P 8}$ & -5.21 & $-10.31 * *$ & $-7.77 * *$ & -4.71 & -4.92 & $-4.81 *$ \\
\hline P6 $\times$ P9 & -1.04 & -4.64 & -2.85 & -0.52 & 1.09 & 0.27 \\
\hline $\mathrm{P} 6 \times \mathbf{P 1 0}$ & -1.04 & 0.52 & -0.26 & -0.52 & $6.56^{*}$ & 2.94 \\
\hline $\mathbf{P 7} \times \mathbf{P 8}$ & 0.00 & $-6.70^{*}$ & -3.37 & 0.52 & -1.09 & -0.27 \\
\hline $\mathbf{P 7} \times \mathbf{P 9}$ & -4.69 & $-9.79 * *$ & $-7.25 * *$ & -4.19 & -4.37 & -4.28 \\
\hline $\mathbf{P 7} \times \mathbf{P 1 0}$ & -0.52 & 1.55 & 0.52 & 0.00 & $7.65^{*}$ & 3.74 \\
\hline $\mathbf{P 8} \times \mathbf{P 9}$ & -4.69 & $-8.76^{* *}$ & $-6.74 * *$ & -4.19 & -3.28 & -3.74 \\
\hline P8 $\times$ P10 & $9.38 * *$ & 5.67 & $7.51 * *$ & $9.95 * *$ & $12.02 * *$ & $10.96^{* *}$ \\
\hline P9 $\times$ P10 & 2.08 & 4.12 & 3.11 & 2.62 & $10.38^{* *}$ & $6.42 * *$ \\
\hline
\end{tabular}

D1, D2 and com., refer to first, second planting dates and combined data, respectively.

$*$ and $* *$ significant at 0.05 and 0.01 levels of probability, respectively. 
Table 4. Heterosis for plant height relative to S.C. 10 and S.C. $30 \mathrm{K8}$ in two planting dates as well as combined data

\begin{tabular}{|c|c|c|c|c|c|c|}
\hline \multirow{3}{*}{$\begin{array}{l}\text { Trait } \\
\text { Cross }\end{array}$} & \multicolumn{6}{|c|}{ Plant height (cm) } \\
\hline & \multicolumn{3}{|c|}{ Heterosis (\%) relative to S.C. 10} & \multicolumn{3}{|c|}{ Heterosis (\%) relative to S.C. 30 K 8} \\
\hline & D1 & D2 & Com. & D1 & D2 & Com. \\
\hline$\overline{\mathbf{P 1} \times \mathbf{P 2}}$ & -3.33 & -3.83 & -3.58 & 1.57 & -1.84 & -0.18 \\
\hline $\mathbf{P 1} \times \mathbf{P 3}$ & $-9.52 *$ & -8.00 & $-8.75 * *$ & -4.94 & -6.09 & -5.53 \\
\hline $\mathbf{P 1} \times \mathbf{P 4}$ & $-8.94 *$ & -9.01 & $-8.98 * *$ & -4.34 & -7.13 & -5.76 \\
\hline P1 $\times$ P5 & -8.72 & -6.64 & $-7.67 *$ & -4.10 & -4.71 & -4.41 \\
\hline $\mathbf{P 1} \times \mathbf{P 6}$ & $-9.17 *$ & -6.76 & $-7.95 *$ & -4.58 & -4.83 & -4.71 \\
\hline $\mathbf{P 1} \times \mathbf{P 7}$ & $-15.94 * *$ & $-12.16^{* *}$ & $-14.03 * *$ & $-11.69 *$ & $-10.34^{*}$ & $-11.00 *$ \\
\hline P1 $\times$ P8 & $-14.91 * *$ & $-27.82 * *$ & $-21.42 * *$ & $-10.60 *$ & $-26.32 * *$ & $-18.65^{*}$ \\
\hline $\mathbf{P 1} \times \mathbf{P 9}$ & $-12.27 * *$ & -4.17 & $-8.18 *$ & -7.83 & -2.18 & -4.94 \\
\hline $\mathbf{P} 1 \times \mathbf{P} 10$ & -2.52 & $-13.85 * *$ & $-8.24 *$ & 2.41 & $-12.07 *$ & -5.00 \\
\hline $\mathbf{P 2} \times \mathbf{P 3}$ & 3.67 & -2.59 & 0.51 & 8.92 & -0.57 & 4.06 \\
\hline $\mathbf{P 2} \times \mathbf{P 4}$ & -0.23 & -3.38 & -1.82 & 4.82 & -1.38 & 1.65 \\
\hline $\mathbf{P 2} \times \mathbf{P 5}$ & 1.61 & -5.86 & -2.16 & 6.75 & -3.91 & 1.29 \\
\hline $\mathbf{P 2} \times \mathbf{P 6}$ & 3.21 & -6.53 & -1.70 & 8.43 & -4.60 & 1.76 \\
\hline $\mathbf{P 2} \times \mathbf{P 7}$ & -0.80 & -8.11 & -4.49 & 4.22 & -6.21 & -1.12 \\
\hline $\mathbf{P 2} \times \mathbf{P 8}$ & $-16.06 * *$ & -7.88 & $-11.93 * *$ & $-11.81 *$ & -5.98 & $-8.82 *$ \\
\hline $\mathbf{P 2} \times \mathbf{P 9}$ & 3.21 & -7.66 & -2.27 & 8.43 & -5.75 & 1.18 \\
\hline $\mathbf{P 2} \times \mathbf{P} 10$ & -1.49 & -8.33 & -4.94 & 3.49 & -6.44 & -1.59 \\
\hline $\mathbf{P 3} \times \mathbf{P 4}$ & 2.18 & -6.64 & -2.27 & 7.35 & -4.71 & 1.18 \\
\hline P3 $\times$ P5 & -2.64 & $-14.41 * *$ & $-8.58 * *$ & 2.29 & $-12.64 * *$ & -5.35 \\
\hline P3 $\times$ P6 & 1.03 & $-15.20 * *$ & $-7.16^{*}$ & 6.14 & $-13.45^{* *}$ & -3.88 \\
\hline $\mathbf{P 3} \times \mathbf{P 7}$ & -1.95 & $-15.09 * *$ & $-8.58 * *$ & 3.01 & $-13.33 * *$ & -5.35 \\
\hline $\mathbf{P 3} \times \mathbf{P 8}$ & -5.28 & $-23.76 * *$ & $-14.60 * *$ & -0.48 & $-22.18 * *$ & $-11.59 *$ \\
\hline $\mathbf{P 3} \times \mathbf{P 9}$ & 1.72 & -8.22 & -3.30 & 6.87 & -6.32 & 0.12 \\
\hline $\mathbf{P 3} \times \mathbf{P 1 0}$ & -0.57 & $-10.47^{*}$ & -5.57 & 4.46 & -8.62 & -2.24 \\
\hline P4 $\times$ P5 & -7.80 & $-17.79 * *$ & $-12.84 * *$ & -3.13 & $-16.09 * *$ & $-9.76 *$ \\
\hline P4 $\times$ P6 & -5.85 & -8.00 & $-6.93 *$ & -1.08 & -6.09 & -3.65 \\
\hline P4 $\times$ P7 & $-8.83^{*}$ & $-9.35 *$ & $-9.09 * *$ & -4.22 & -7.47 & -5.88 \\
\hline $\mathbf{P 4} \times \mathbf{P 8}$ & $-10.89 *$ & $-12.73 * *$ & $-11.82 * *$ & -6.39 & $-10.92 *$ & $-8.71 *$ \\
\hline P4 $\times$ P9 & -3.67 & $-11.49 *$ & $-7.61 *$ & 1.20 & $-9.66^{*}$ & -4.35 \\
\hline P4 $\times$ P10 & $-10.21 *$ & $-17.12 * *$ & $-13.69 * *$ & -5.66 & $-15.40 * *$ & $-10.65^{*}$ \\
\hline $\mathbf{P 5} \times \mathbf{P 6}$ & -7.91 & $-15.43 * *$ & $-11.70 * *$ & -3.25 & $-13.68 * *$ & $-8.59 *$ \\
\hline P5 $\times$ P7 & $-22.59 * *$ & $-17.68 * *$ & $-20.11 * *$ & $-18.67 * *$ & $-15.98 * *$ & $-17.29 *$ \\
\hline P5 $\times$ P8 & -3.67 & $-12.84 * *$ & $-8.30 *$ & 1.20 & $-11.03 *$ & -5.06 \\
\hline $\mathbf{P 5} \times \mathbf{P 9}$ & -1.83 & $-17.12 * *$ & $-9.55 * *$ & 3.13 & $-15.40 * *$ & -6.35 \\
\hline P5 $\times$ P10 & -7.80 & $-18.47 * *$ & $-13.18 * *$ & -3.13 & $-16.78 * *$ & $-10.12 *$ \\
\hline $\mathbf{P 6} \times \mathbf{P 7}$ & -5.39 & $-13.85 * *$ & $-9.66 * *$ & -0.60 & $-12.07^{*}$ & $-6.47^{*}$ \\
\hline P6 $\times$ P8 & -5.39 & $-10.81 *$ & $-8.13^{*}$ & -0.60 & -8.97 & -4.88 \\
\hline P6 $\times$ P9 & -1.72 & -8.56 & -5.17 & 3.25 & -6.67 & -1.82 \\
\hline P6 $\times$ P10 & -3.56 & $-12.05 * *$ & $-7.84 *$ & 1.33 & $-10.23^{*}$ & -4.59 \\
\hline P7 $\times$ P8 & -7.45 & $-14.19 * *$ & $-10.85^{* *}$ & -2.77 & $-12.41 * *$ & $-7.71 *$ \\
\hline P7 $\times$ P9 & -4.13 & $-10.47^{*}$ & $-7.33 *$ & 0.72 & -8.62 & -4.06 \\
\hline $\mathbf{P 7} \times \mathbf{P 1 0}$ & $-11.01 *$ & $-14.64 * *$ & $-12.84 * *$ & -6.51 & $-12.87 * *$ & $-9.76^{*}$ \\
\hline P8 $\times$ P9 & -4.01 & -8.56 & $-6.31 *$ & 0.84 & -6.67 & -3.00 \\
\hline $\mathbf{P 8} \times \mathbf{P 1 0}$ & $-11.93 * *$ & $-21.62 * *$ & $-16.82 * *$ & -7.47 & $-20.00 * *$ & $-13.88 *$ \\
\hline $\mathrm{P} 9 \times \mathrm{P} 10$ & -5.96 & $-20.83 * *$ & $-13.47 * *$ & -1.20 & $-19.20 * *$ & $-10.41 *$ \\
\hline
\end{tabular}

D1 , D2 and com. refer to first, second planting dates and combined data, respectively.

$*$ and $* *$ significant at 0.05 and 0.01 levels of probability, respectively. 
Table 5. Heterosis for ear height relative to S.C. 10 and S.C. $30 \mathrm{K8}$ in two planting dates as well as combined data

\begin{tabular}{|c|c|c|c|c|c|c|}
\hline \multirow{3}{*}{$\begin{array}{l}\text { Trait } \\
\text { Cross }\end{array}$} & \multicolumn{6}{|c|}{ Ear height (cm) } \\
\hline & \multicolumn{3}{|c|}{ Heterosis (\%) relative to S.C. 10} & \multicolumn{3}{|c|}{ Heterosis (\%) relative to S.C. $30 \mathrm{~K} 8$} \\
\hline & D1 & D2 & Com. & D1 & $\mathbf{D 2}$ & Com. \\
\hline$\overline{\mathbf{P 1} \times \mathbf{P 2}}$ & 0.22 & $-9.09 *$ & -4.45 & 7.67 & -0.97 & 3.37 \\
\hline $\mathbf{P 1} \times \mathbf{P 3}$ & -5.58 & $-11.53 * *$ & $-8.57 * *$ & 1.44 & -3.62 & -1.08 \\
\hline $\mathbf{P 1} \times \mathbf{P 4}$ & $-12.50 * *$ & $-12.86^{* *}$ & $-12.68 * *$ & -6.00 & -5.07 & -5.54 \\
\hline P1 $\times$ P5 & $-11.16^{*}$ & $-10.20 *$ & $-10.68 * *$ & -4.56 & -2.17 & -3.37 \\
\hline $\mathbf{P 1} \times \mathbf{P 6}$ & $-10.04 *$ & -7.76 & $-8.90 * *$ & -3.36 & 0.48 & -1.44 \\
\hline $\mathbf{P 1} \times \mathbf{P 7}$ & $-18.53 * *$ & $-11.97 * *$ & $-15.24 * *$ & $-12.47^{*}$ & -4.11 & $-8.30 *$ \\
\hline P1 $\times$ P8 & $-15.63 * *$ & $-26.83 * *$ & $-21.25 * *$ & -9.35 & $-20.29 * *$ & $-14.80 * *$ \\
\hline $\mathbf{P 1} \times \mathbf{P 9}$ & $-11.16^{*}$ & -7.98 & $-9.57 * *$ & -4.56 & 0.24 & -2.17 \\
\hline $\mathbf{P} 1 \times \mathbf{P} 10$ & -8.04 & $-9.09 *$ & $-8.57 * *$ & -1.20 & -0.97 & -1.08 \\
\hline $\mathbf{P 2} \times \mathbf{P 3}$ & -6.70 & -0.44 & -3.56 & 0.24 & 8.45 & 4.33 \\
\hline $\mathbf{P 2} \times \mathbf{P 4}$ & 1.12 & -4.88 & -1.89 & 8.63 & 3.62 & 6.14 \\
\hline $\mathbf{P 2} \times \mathbf{P 5}$ & 0.67 & -1.33 & -0.33 & 8.15 & 7.49 & $7.82 *$ \\
\hline $\mathbf{P 2} \times \mathbf{P 6}$ & 6.92 & -3.55 & 1.67 & $14.87 * *$ & 5.07 & $9.99 * *$ \\
\hline $\mathbf{P 2} \times \mathbf{P 7}$ & 7.14 & -7.54 & -0.22 & $15.11 * *$ & 0.72 & $7.94 *$ \\
\hline $\mathbf{P 2} \times \mathbf{P 8}$ & -1.34 & $-11.53 * *$ & $-6.45 *$ & 6.00 & -3.62 & 1.20 \\
\hline $\mathbf{P 2} \times \mathbf{P 9}$ & 8.04 & -8.43 & -0.22 & $16.07 * *$ & -0.24 & $7.94 *$ \\
\hline $\mathbf{P 2} \times \mathbf{P 1 0}$ & 7.59 & -5.54 & 1.00 & $15.59 * *$ & 2.90 & $9.27 * *$ \\
\hline P3 $\times$ P4 & 0.22 & $-9.31 *$ & -4.56 & 7.67 & -1.21 & 3.25 \\
\hline P3 $\times$ P5 & -1.12 & $-15.30 * *$ & $-8.23 *$ & 6.24 & -7.73 & -0.72 \\
\hline $\mathbf{P 3} \times \mathbf{P 6}$ & -2.68 & $-14.63 * *$ & $-8.68 * *$ & 4.56 & -7.00 & -1.20 \\
\hline $\mathbf{P 3} \times \mathbf{P 7}$ & 0.22 & $-11.53 * *$ & -5.67 & 7.67 & -3.62 & 2.05 \\
\hline $\mathbf{P 3} \times \mathbf{P 8}$ & -1.56 & $-24.17 * *$ & $-12.90 * *$ & 5.76 & $-17.39 * *$ & -5.78 \\
\hline $\mathbf{P 3} \times \mathbf{P 9}$ & 4.69 & -5.10 & -0.22 & $12.47 *$ & 3.38 & $7.94 *$ \\
\hline $\mathbf{P 3} \times \mathbf{P 1 0}$ & -4.46 & $-9.98 *$ & $-7.23 *$ & 2.64 & -1.93 & 0.36 \\
\hline P4 $\times$ P5 & -3.57 & $-13.30 * *$ & $-8.45 * *$ & 3.60 & -5.56 & -0.96 \\
\hline $\mathbf{P 4} \times \mathbf{P 6}$ & -8.26 & $-17.29 * *$ & $-12.79 * *$ & -1.44 & $-9.90 *$ & -5.66 \\
\hline $\mathbf{P 4} \times \mathbf{P 7}$ & $-11.38^{*}$ & $-13.53 * *$ & $-12.46^{* *}$ & -4.80 & -5.80 & -5.29 \\
\hline P4 $\times$ P8 & $-15.85 * *$ & $-12.64 * *$ & $-14.24 * *$ & -9.59 & -4.83 & $-7.22 *$ \\
\hline $\mathbf{P 4} \times \mathbf{P 9}$ & -5.58 & $-12.64 * *$ & $-9.12 * *$ & 1.44 & -4.83 & -1.68 \\
\hline P4 $\times$ P10 & $-12.50 * *$ & $-13.53 * *$ & $-13.01 * *$ & -6.00 & -5.80 & -5.90 \\
\hline P5 $\times$ P6 & $-12.28 *$ & $-14.19 * *$ & $-13.24 * *$ & -5.76 & -6.52 & -6.14 \\
\hline P5 $\times \mathbf{P 7}$ & $-19.87 * *$ & $-17.07 * *$ & $-18.46^{* *}$ & $-13.91 * *$ & $-9.66^{*}$ & $-11.79 * *$ \\
\hline P5 $\times$ P8 & -6.47 & $-16.19 * *$ & $-11.35 * *$ & 0.48 & -8.70 & -4.09 \\
\hline P5 $\times$ P9 & $-11.83 *$ & $-16.41 * *$ & $-14.13 * *$ & -5.28 & -8.94 & $-7.10 *$ \\
\hline P5 $\times$ P10 & -6.25 & $-16.85 * *$ & $-11.57 * *$ & 0.72 & $-9.42 *$ & -4.33 \\
\hline P6 $\times$ P7 & -5.36 & $-23.50 * *$ & $-14.46^{* *}$ & 1.68 & $-16.67 * *$ & $-7.46^{*}$ \\
\hline P6 $\times$ P8 & -8.04 & $-10.64 *$ & $-9.34 * *$ & -1.20 & -2.66 & -1.93 \\
\hline $\mathbf{P 6} \times \mathbf{P 9}$ & -9.15 & $-10.86 *$ & $-10.01 * *$ & -2.40 & -2.90 & -2.65 \\
\hline $\mathrm{P} 6 \times \mathrm{P} 10$ & -4.46 & $-10.42 *$ & $-7.45^{*}$ & 2.64 & -2.42 & 0.12 \\
\hline $\mathbf{P 7} \times \mathbf{P 8}$ & $-9.82 *$ & $-13.30 * *$ & $-11.57 * *$ & -3.12 & -5.56 & -4.33 \\
\hline $\mathbf{P 7} \times \mathbf{P 9}$ & -7.37 & $-13.30 * *$ & $-10.34 * *$ & -0.48 & -5.56 & -3.01 \\
\hline $\mathbf{P 7} \times \mathbf{P} 10$ & -8.93 & $-14.63 * *$ & $-11.79 * *$ & -2.16 & -7.00 & -4.57 \\
\hline $\mathbf{P 8} \times \mathbf{P 9}$ & -6.70 & $-10.42 *$ & $-8.57 * *$ & 0.24 & -2.42 & -1.08 \\
\hline $\mathbf{P 8} \times \mathbf{P 1 0}$ & $-13.62 * *$ & $-18.18 * *$ & $-15.91 * *$ & -7.19 & $-10.87 *$ & $-9.03 *$ \\
\hline $\mathbf{P 9} \times \mathbf{P 1 0}$ & -8.71 & $-16.85^{* *}$ & $-12.79 * *$ & -1.92 & $-9.42 *$ & -5.66 \\
\hline
\end{tabular}

D1 , D2 and com. refer to first, second planting dates and combined data, respectively.

$*$ and ${ }^{* *}$ significant at 0.05 and 0.01 levels of probability, respectively. 
Table 6. Heterosis for chlorophyll content relative to S.C. 10 and S.C. 30 K8 in two planting dates as well as combined data

\begin{tabular}{|c|c|c|c|c|c|c|}
\hline \multirow{3}{*}{$\begin{array}{l}\text { Trait } \\
\text { Cross } \\
\end{array}$} & \multicolumn{6}{|c|}{ Chlorophyll content } \\
\hline & \multicolumn{3}{|c|}{ Heterosis (\%) relative to S.C. 10} & \multicolumn{3}{|c|}{ Heterosis (\%) relative to S.C. 30 K 8} \\
\hline & D1 & D2 & Com. & D1 & D2 & Com. \\
\hline$\overline{\mathbf{P 1} \times \mathbf{P 2}}$ & 5.72 & 9.59 & $7.57^{*}$ & 2.20 & 4.26 & 3.20 \\
\hline $\mathbf{P 1} \times \mathbf{P 3}$ & 4.31 & -3.64 & 0.49 & 0.84 & -8.32 & -3.59 \\
\hline P1 $\times$ P4 & -4.73 & -0.64 & -2.77 & -7.90 & -5.47 & -6.72 \\
\hline P1 $\times$ P5 & -1.57 & 11.09 & 4.51 & -4.84 & 5.70 & 0.26 \\
\hline $\mathbf{P 1} \times \mathbf{P 6}$ & $-11.24 *$ & -0.78 & -6.22 & $-14.20 * *$ & -5.60 & $-10.04 * *$ \\
\hline $\mathbf{P 1} \times \mathbf{P 7}$ & $-20.71 * *$ & 4.40 & $-8.66^{*}$ & $-23.35 * *$ & -0.67 & $-12.38^{* *}$ \\
\hline $\mathbf{P 1} \times \mathbf{P 8}$ & $-14.97 * *$ & 8.56 & -3.68 & $-17.80 * *$ & 3.29 & $-7.60 *$ \\
\hline $\mathbf{P 1} \times \mathbf{P 9}$ & $-23.02 * *$ & -5.87 & $-14.79 * *$ & $-25.58 * *$ & -10.44 & $-18.26^{* *}$ \\
\hline $\mathbf{P} 1 \times \mathbf{P} 10$ & -5.99 & 2.00 & -2.16 & $-9.12 *$ & -2.96 & -6.14 \\
\hline $\mathbf{P 2} \times \mathbf{P 3}$ & 8.50 & $12.74 *$ & $10.54 * *$ & 4.89 & 7.26 & 6.04 \\
\hline $\mathbf{P 2} \times \mathbf{P 4}$ & $12.87 * *$ & 11.40 & $12.17 * *$ & $9.12 *$ & 5.99 & $7.60^{*}$ \\
\hline $\mathbf{P 2} \times \mathbf{P 5}$ & $8.91 *$ & 11.54 & $10.17^{* *}$ & 5.28 & 6.12 & 5.69 \\
\hline $\mathbf{P 2} \times \mathbf{P 6}$ & $11.70 * *$ & 10.84 & $11.28 * *$ & 7.98 & 5.45 & 6.76 \\
\hline $\mathbf{P} 2 \times \mathbf{P} 7$ & $12.39 * *$ & $15.86^{*}$ & $14.06^{* *}$ & $8.65^{*}$ & 10.24 & $9.42 * *$ \\
\hline $\mathbf{P 2} \times \mathbf{P 8}$ & $14.05 * *$ & $18.33 * *$ & $16.11 * *$ & $10.26^{*}$ & $12.59 *$ & $11.38 * *$ \\
\hline $\mathbf{P 2} \times \mathbf{P 9}$ & 7.90 & $16.44 * *$ & $12.00 * *$ & 4.31 & 10.79 & $7.44 *$ \\
\hline $\mathbf{P} 2 \times \mathbf{P} 10$ & 7.95 & $17.72 * *$ & $12.64 * *$ & 4.35 & $12.01 *$ & $8.06^{*}$ \\
\hline P3 $\times$ P4 & 1.22 & 4.48 & 2.78 & -2.15 & -0.59 & -1.40 \\
\hline P3 $\times$ P5 & -4.23 & -8.73 & -6.39 & -7.42 & $-13.17 *$ & $-10.20 * *$ \\
\hline $\mathbf{P 3} \times \mathbf{P 6}$ & -0.33 & 4.91 & 2.18 & -3.65 & -0.19 & -1.97 \\
\hline P3 $\times \mathbf{P 7}$ & 0.89 & 5.04 & 2.88 & -2.47 & -0.06 & -1.30 \\
\hline P3 $\times$ P8 & 1.89 & 1.16 & 1.54 & -1.50 & -3.76 & -2.59 \\
\hline P3 $\times$ P9 & 3.73 & 4.57 & 4.13 & 0.28 & -0.51 & -0.10 \\
\hline $\mathbf{P 3} \times \mathbf{P 1 0}$ & 2.38 & 10.52 & 6.28 & -1.03 & 5.15 & 1.96 \\
\hline P4 $\times$ P5 & 4.64 & 5.09 & 4.85 & 1.15 & -0.01 & 0.59 \\
\hline $\mathbf{P 4} \times \mathbf{P 6}$ & 2.71 & 4.59 & 3.61 & -0.71 & -0.49 & -0.61 \\
\hline P4 $\times$ P7 & -3.99 & 5.94 & 0.77 & -7.19 & 0.80 & -3.32 \\
\hline P4 $\times$ P8 & -4.32 & 3.35 & -0.64 & -7.51 & -1.67 & -4.68 \\
\hline $\mathbf{P 4} \times \mathbf{P 9}$ & -8.44 & 1.86 & -3.50 & $-11.49 * *$ & -3.09 & $-7.42 *$ \\
\hline $\mathbf{P} 4 \times \mathbf{P} 10$ & 0.52 & $12.25^{*}$ & 6.15 & -2.83 & 6.80 & 1.83 \\
\hline $\mathrm{P5} \times \mathrm{P6}$ & $14.36^{* *}$ & $27.38 * *$ & $20.61^{* *}$ & $10.56^{*}$ & $21.20 * *$ & $15.70 * *$ \\
\hline $\mathbf{P 5} \times \mathbf{P 7}$ & -3.79 & 2.96 & -0.56 & -7.00 & -2.04 & -4.60 \\
\hline $\mathrm{P} 5 \times \mathrm{P} 8$ & -0.15 & 3.89 & 1.79 & -3.47 & -1.16 & -2.35 \\
\hline P5 $\times$ P9 & -7.77 & 6.22 & -1.06 & $-10.84 *$ & 1.06 & -5.08 \\
\hline $\mathrm{P} 5 \times \mathrm{P} 10$ & 2.93 & 8.06 & 5.39 & -0.50 & 2.81 & 1.10 \\
\hline P6 $\times$ P7 & $-10.55^{*}$ & -2.07 & -6.48 & $-13.53 * *$ & -6.83 & $-10.29 * *$ \\
\hline P6 $\times$ P8 & $13.94 * *$ & $19.06^{* *}$ & $16.40^{* *}$ & $10.15^{*}$ & $13.28 *$ & $11.66^{* *}$ \\
\hline $\mathbf{P 6} \times \mathbf{P 9}$ & $-12.16 * *$ & -1.98 & $-7.28 *$ & $-15.09 * *$ & -6.74 & $-11.05^{* *}$ \\
\hline P6 $\times$ P10 & $13.41^{* *}$ & $19.31 * *$ & $16.24 * *$ & $9.63 *$ & $13.51^{*}$ & $11.51^{* *}$ \\
\hline $\mathbf{P 7} \times \mathbf{P 8}$ & -5.66 & 4.89 & -0.60 & $-8.80 *$ & -0.20 & -4.64 \\
\hline $\mathbf{P 7} \times \mathbf{P 9}$ & $-12.53 * *$ & 2.64 & -5.25 & $-15.44 * *$ & -2.35 & $-9.11 *$ \\
\hline $\mathbf{P} 7 \times \mathbf{P} 10$ & -2.12 & 1.23 & -0.51 & -5.38 & -3.68 & -4.56 \\
\hline $\mathrm{P8} \times \mathrm{P9}$ & -6.31 & 1.81 & -2.41 & $-9.43 *$ & -3.13 & -6.38 \\
\hline $\mathrm{P} 8 \times \mathrm{P} 10$ & -6.83 & 0.08 & -3.52 & $-9.93 *$ & -4.78 & $-7.44 *$ \\
\hline $\mathrm{P9} \times \mathrm{P} 10$ & 1.14 & 0.78 & 0.97 & -2.23 & -4.12 & -3.14 \\
\hline
\end{tabular}

D1 , D2 and com. refer to first, second planting dates and combined data, respectively.

* and ** significant at 0.05 and 0.01 levels of probability, respectively. 


\section{Grain yield/ plant}

Standard heterosis effects for grain yield/ plant relative to S.C. 10 and S.C. $30 \mathrm{k} 8$ in both planting dates as well as combined analysis are presented in Table 7. Results indicate that three, four and four crosses expressed significant and positive standard heterosis relative to S.C. 10 in the first, second planting dates as well as combined analysis, respectively. Three single crosses exhibited significant and positive heterotic effects relative to S.C. $30 \mathrm{k} 8$, in the respective case. However, the cross combination $\mathrm{P}_{6} \times \mathrm{P}_{8}$ gave the most desirable heterotic effects relative to S.C. 10 recording $17.98 ; 22.81$ and $20.10 \%$ in the first, second planting dates and combined data, respectively. The respective values for this cross relative to S.C. $30 \mathrm{k} 8$ were $17.18,18.39$ and $17.72 \%$. Moreover, the cross $\mathrm{P}_{6} \times \mathrm{P}_{10}$ ranked the second best for heterosis relative to S.C. $10(19.23 \%)$ and S.C. $30 \mathrm{k} 8$ $(16.87 \%)$ in the combined data. Also, the cross $\mathrm{P}_{5} \times \mathrm{P}_{6}$ ranked the third best recording heterotic values of 18.17 and $15.83 \%$ relative to S.C. 10 and S.C. $30 \mathrm{k} 8$, respectively in the combined analysis. Similar results were reported by Sedhom et al. (2012), El-Badawy (2013), AbdElaziz (2014), Abdel-Moneam et al. (2014), Zaid et al. (2014), Ram et al. (2015) and Reddy et al. (2015).

\section{Quality traits}

Standard heterosis effects for protein (\%) and oil (\%) relative to S.C. 10 and S.C. $30 \mathrm{k} 8$ in both planting dates as well as combined analysis are presented in Tables 8 and 9. Results indicate that heterosis effects for protein (\%) relative to S.C. 10 ranged from -18.80 to $22.02,-18.06$ to 18.00 and -16.48 to $19.12 \%$ in the first, second planting dates and combined analyses, respectively. The respective values of heterotic effects relative to S.C. $30 \mathrm{k} 8$ ranged from -24.74 to $13.10,-19.53$ to 15.89 and -20.34 to $13.62 \%$. However, the single cross $\mathrm{P}_{2} \times \mathrm{P}_{8}$ gave the most desirable heterotic effects in the first planting date recording $22.02 \%$ and $13.10 \%$ relative to S.C. 10 and S.C. $30 \mathrm{k} 8$, respectively (Table 8). Meantime, the cross $\mathrm{P}_{4} \times \mathrm{P}_{7}$ expressed the highest positive and significant heterotic effects for protein (\%) recording 18.00 and $19.12 \%$ relative to S.C. 10 and 15.89 and $13.62 \%$ relative to S.C. $30 \mathrm{k} 8$, in the second planting date and combined analyses, respectively.
In regard to oil content, three, five and one crosses exhibited positive and significant heterotic effects relative to S.C. 10 in the first, second planting dates well as combined data, respectively. One, four and one cross expressed desirable heterotic effects relative to S.C. $30 \mathrm{k} 8$ in the three respective cases. However, the cross combination $\mathrm{P}_{1} \times \mathrm{P}_{2}$ gave the most desirable standard heterosis for oil (\%) in the combined analysis $(20.02 \%)$ relative to S.C. 10 and $(16.99 \%)$ relative to SC $30 \mathrm{k} 8$. While, the cross $\mathrm{P}_{1} \times \mathrm{P}_{4}$ gave the best heterotic effects for this trait in the second planting date being $30.11 \%$ relative to SC 10 and $28.46 \%$ relative to S.C. 30 k8 (Table 9). These results are in agreement with those obtained by Bekele and Rao (2013), Lahane et al. (2014) and Ulaganathan and Ibrahim (2014).

From such results, it could be concluded that the single crosses $\mathrm{P}_{5} \times \mathrm{P}_{6}, \mathrm{P}_{6} \times \mathrm{P}_{8}$ and $\mathrm{P}_{6} \times \mathrm{P}_{10}$ are promising and could be used for improving grain yield of maize.

\section{Correlation coefficient and factor analysis}

Correlation coefficients between grain yield/ plant and days to $50 \%$ tasseling, days to $50 \%$ silking, plant height, ear height, chlorophyll content, ear length, ear diameter, number of rows, number of grains/row, 100 grain weight and shelling (\%) in the combined data are presented in Table 10. Results indicate that positive and highly significant correlation coefficient values were registered between grain yield/plant and each of chlorophyll content $\left(0.6372^{* *}\right)$, ear length $\left(0.5116^{* *}\right)$, ear diameter $(0.6074 * *)$, number of rows/ear $(0.4794 * *)$, number of grains/row $\left(0.6909^{* *}\right), 100$ grain weight $\left(0.7344^{* *}\right)$ and shelling $(\%)\left(0.4021^{* *}\right)$. The association between days to $50 \%$ tasseling and days to $50 \%$ silking $\left(0.9406^{* *}\right)$ was positive and highly significant. Also, the correlation coefficient between plant height and each of ear height $\left(0.8512^{* *}\right)$ and chlorophyll content $\left(0.2993^{*}\right)$ was positive and significant. Also, the correlation between ear height and chlorophyll content $\left(0.4855^{* *}\right)$ was positive and highly significant. The association between chlorophyll content and each of number of rows/ear $\left(0.5134^{* *}\right)$, number of grains/row $\left(0.4235^{* *}\right)$, 100 grain weight $\left(0.4435^{* *}\right)$ and shelling (\%) $\left(0.3575^{*}\right)$ was positive and significant. 
Table 7. Heterosis for grain yield/ plant relative to S.C. 10 and S.C. $30 \mathrm{K8}$ in two planting dates as well as combined data

\begin{tabular}{|c|c|c|c|c|c|c|}
\hline \multirow{3}{*}{$\begin{array}{l}\text { Trait } \\
\text { Cross } \\
\end{array}$} & \multicolumn{6}{|c|}{ Grain yield/ plant (g) } \\
\hline & \multicolumn{3}{|c|}{ Heterosis (\%) relative to S.C. 10} & \multicolumn{3}{|c|}{ Heterosis (\%) relative to S.C. 30 K 8} \\
\hline & D1 & D2 & Com. & D1 & D2 & Com. \\
\hline$\overline{\mathbf{P 1} \times \mathbf{P 2}}$ & -8.90 & -7.02 & -8.08 & -9.52 & -10.36 & -9.90 \\
\hline $\mathbf{P 1} \times \mathbf{P 3}$ & -15.07 & 1.54 & -7.79 & -15.65 & -2.11 & -9.61 \\
\hline P1 × P4 & -10.96 & $-19.08^{*}$ & $-14.52 *$ & -11.56 & $-21.99 *$ & $-16.21 * *$ \\
\hline P1 $\times$ P5 & -4.28 & 2.63 & -1.25 & -4.93 & -1.06 & -3.20 \\
\hline $\mathbf{P 1} \times \mathbf{P 6}$ & -8.90 & -3.51 & -6.54 & -9.52 & -6.98 & -8.39 \\
\hline $\mathbf{P 1} \times \mathbf{P 7}$ & $-22.60 * *$ & -8.99 & $-16.63^{* *}$ & $-23.13^{* *}$ & -12.26 & $-18.28 * *$ \\
\hline $\mathbf{P 1} \times \mathbf{P 8}$ & $-23.46^{* *}$ & -5.92 & $-15.77 * *$ & $-23.98 * *$ & -9.30 & $-17.44 * *$ \\
\hline $\mathbf{P 1} \times \mathbf{P 9}$ & $-30.82 * *$ & -12.50 & $-22.79 * *$ & $-31.29 * *$ & -15.64 & $-24.32 * *$ \\
\hline $\mathbf{P} 1 \times \mathbf{P} 10$ & $-17.29^{*}$ & -5.92 & $-12.31^{*}$ & $-17.86^{*}$ & -9.30 & $-14.04 *$ \\
\hline $\mathbf{P 2} \times \mathbf{P 3}$ & $-43.15^{* *}$ & -16.89 & $-31.63 * *$ & $-43.54 * *$ & $-19.87^{*}$ & $-32.99 * *$ \\
\hline P2 $\times \mathbf{P 4}$ & -11.30 & 8.11 & -2.79 & -11.90 & 4.23 & -4.71 \\
\hline $\mathbf{P 2} \times \mathbf{P 5}$ & -3.42 & 12.72 & 3.65 & -4.08 & 8.67 & 1.60 \\
\hline $\mathbf{P 2} \times \mathbf{P 6}$ & -4.28 & 5.26 & -0.10 & -4.93 & 1.48 & -2.07 \\
\hline $\mathbf{P} 2 \times \mathbf{P} 7$ & -7.88 & -1.97 & -5.29 & -8.50 & -5.50 & -7.16 \\
\hline $\mathbf{P} 2 \times \mathbf{P 8}$ & 5.82 & $21.93 *$ & $12.88^{*}$ & 5.10 & $17.55^{*}$ & 10.65 \\
\hline $\mathbf{P 2} \times \mathbf{P 9}$ & -11.99 & 8.33 & -3.08 & -12.59 & 4.44 & -5.00 \\
\hline $\mathbf{P} 2 \times \mathbf{P} 10$ & -3.08 & 16.01 & 5.29 & -3.74 & 11.84 & 3.20 \\
\hline P3 $\times$ P4 & -11.47 & -3.95 & -8.17 & -12.07 & -7.40 & -9.99 \\
\hline P3 $\times$ P5 & $-16.44 *$ & -6.80 & $-12.21^{*}$ & $-17.01^{*}$ & -10.15 & $-13.95^{*}$ \\
\hline $\mathbf{P 3} \times \mathbf{P 6}$ & -2.57 & 12.72 & 4.13 & -3.23 & 8.67 & 2.07 \\
\hline $\mathbf{P 3} \times \mathbf{P 7}$ & -15.75 & -16.89 & $-16.25^{* *}$ & $-16.33 *$ & $-19.87 *$ & $-17.91 * *$ \\
\hline $\mathbf{P 3} \times \mathbf{P 8}$ & -4.11 & -14.04 & -8.46 & -4.76 & $-17.12^{*}$ & -10.27 \\
\hline $\mathbf{P 3} \times \mathbf{P 9}$ & -15.41 & -15.57 & $-15.48^{*}$ & $-15.99 *$ & $-18.60 *$ & $-17.15^{* *}$ \\
\hline $\mathbf{P 3} \times \mathbf{P 1 0}$ & $-31.16^{* *}$ & -6.14 & $-20.19 * *$ & $-31.63 * *$ & -9.51 & $-21.77 * *$ \\
\hline P4 $\times$ P5 & -3.42 & -16.23 & -9.04 & -4.08 & $-19.24 *$ & -10.84 \\
\hline P4 × P6 & -2.91 & $-23.25^{*}$ & $-11.83 *$ & -3.57 & $-26.00 * *$ & $-13.57^{*}$ \\
\hline P4 $\times$ P7 & -9.59 & -14.91 & $-11.92 *$ & -10.20 & $-17.97 *$ & $-13.67 *$ \\
\hline P4 × P8 & -3.60 & -16.89 & -9.42 & -4.25 & $-19.87 *$ & -11.22 \\
\hline P4 × P9 & -4.79 & -9.21 & -6.73 & -5.44 & -12.47 & -8.58 \\
\hline$P 4 \times P 10$ & -4.97 & $-23.46^{* *}$ & $-13.08 *$ & -5.61 & $-26.22 * *$ & $-14.80 *$ \\
\hline $\mathbf{P 5} \times \mathbf{P 6}$ & $16.95^{*}$ & $19.74 *$ & $18.17 * *$ & $16.16^{*}$ & 15.43 & $15.83 * *$ \\
\hline P5 $\times \mathbf{P 7}$ & -8.90 & -14.69 & -11.44 & -9.52 & $-17.76^{*}$ & $-13.20 *$ \\
\hline $\mathbf{P 5} \times \mathbf{P 8}$ & 3.94 & 10.09 & 6.63 & 3.23 & 6.13 & 4.52 \\
\hline P5 $\times$ P9 & -5.31 & $-26.75^{* *}$ & $-14.71 *$ & -5.95 & $-29.39 * *$ & $-16.40^{* *}$ \\
\hline P5 $\times$ P10 & 4.28 & -9.21 & -1.63 & 3.57 & -12.47 & -3.58 \\
\hline $\mathbf{P 6} \times \mathbf{P 7}$ & -2.57 & -9.87 & -5.77 & -3.23 & -13.11 & -7.63 \\
\hline $\mathbf{P 6} \times \mathbf{P 8}$ & $17.98 *$ & $22.81^{*}$ & $20.10^{* *}$ & $17.18^{*}$ & $18.39 *$ & $17.72 * *$ \\
\hline $\mathbf{P 6} \times \mathbf{P 9}$ & $-29.45^{* *}$ & $-26.32 * *$ & $-28.08 * *$ & $-29.93 * *$ & $-28.96 * *$ & $-29.50^{* *}$ \\
\hline P6 $\times$ P10 & $16.95^{*}$ & $22.15^{*}$ & $19.23 * *$ & $16.16^{*}$ & $17.76^{*}$ & $16.87 * *$ \\
\hline $\mathbf{P 7} \times \mathbf{P 8}$ & -14.73 & -11.84 & $-13.46^{*}$ & -15.31 & -15.01 & $-15.17 *$ \\
\hline P7 $\times$ P9 & $-23.46^{* *}$ & $-23.46 * *$ & $-23.46^{* *}$ & $-23.98 * *$ & $-26.22 * *$ & $-24.98^{* *}$ \\
\hline $\mathbf{P 7} \times \mathbf{P 1 0}$ & -5.48 & 0.88 & -2.69 & -6.12 & -2.75 & -4.62 \\
\hline $\mathbf{P 8} \times \mathbf{P 9}$ & $-17.81 *$ & -7.24 & $-13.17 *$ & $-18.37 *$ & -10.57 & $-14.89 *$ \\
\hline $\mathbf{P 8} \times \mathbf{P 1 0}$ & -11.82 & -7.89 & -10.10 & -12.41 & -11.21 & $-11.88^{*}$ \\
\hline P9 $\times$ P10 & -3.25 & 3.07 & -0.48 & -3.91 & -0.63 & -2.45 \\
\hline
\end{tabular}

D1 , D2 and com. refer to first, second planting dates and combined data, respectively.

* and ** significant at 0.05 and 0.01 levels of probability, respectively. 
Table 8. Heterosis for protein (\%) relative to S.C. 10 and S.C. $30 \mathrm{K8}$ in two planting dates as well as combined data

\begin{tabular}{|c|c|c|c|c|c|c|}
\hline \multirow{3}{*}{$\begin{array}{l}\text { Trait } \\
\text { Cross } \\
\end{array}$} & \multicolumn{6}{|c|}{ Protein (\%) } \\
\hline & \multicolumn{3}{|c|}{ Heterosis (\%) relative to S.C. 10} & \multicolumn{3}{|c|}{ Heterosis (\%) relative to S.C. $30 \mathrm{~K} 8$} \\
\hline & D1 & D2 & Com. & D1 & D2 & Com. \\
\hline$\overline{\mathbf{P 1} \times \mathbf{P 2}}$ & $12.68 * *$ & -5.97 & 3.31 & 4.44 & $-7.65^{*}$ & -1.46 \\
\hline $\mathbf{P 1} \times \mathbf{P 3}$ & 0.15 & -1.97 & -0.91 & -7.18 & -3.72 & $-5.49 *$ \\
\hline P1 $\times$ P4 & $18.50^{* *}$ & 1.73 & $10.08^{* *}$ & 9.83* & -0.09 & $4.99 *$ \\
\hline P1 $\times$ P5 & $14.07 * *$ & -3.40 & $5.29 *$ & 5.72 & -5.13 & 0.43 \\
\hline$P 1 \times P 6$ & $21.09 * *$ & $10.30 * *$ & $15.67 * *$ & $12.23 * *$ & $8.33 * *$ & $10.33 * *$ \\
\hline $\mathbf{P 1} \times \mathbf{P 7}$ & -0.75 & $-9.97 * *$ & $-5.38^{*}$ & $-8.01 *$ & $-11.58 * *$ & $-9.75^{* *}$ \\
\hline $\mathbf{P 1} \times \mathbf{P 8}$ & $-8.83 *$ & 5.70 & -1.53 & $-15.50 * *$ & 3.81 & $-6.08^{*}$ \\
\hline $\mathbf{P 1} \times \mathbf{P 9}$ & 2.68 & -2.27 & 0.19 & -4.83 & -4.02 & -4.43 \\
\hline $\mathbf{P} 1 \times \mathbf{P} 10$ & $14.46^{* *}$ & 1.73 & $8.07 * *$ & 6.09 & -0.09 & 3.07 \\
\hline $\mathbf{P 2} \times \mathbf{P 3}$ & $12.96^{* *}$ & $10.00 * *$ & $11.47 * *$ & 4.69 & $8.03 *$ & $6.32 *$ \\
\hline $\mathbf{P 2} \times \mathbf{P 4}$ & $12.77 * *$ & -1.37 & $5.67 *$ & 4.52 & -3.14 & 0.79 \\
\hline $\mathbf{P 2} \times \mathbf{P 5}$ & $14.07 * *$ & -2.99 & $5.50 *$ & 5.72 & -4.72 & 0.63 \\
\hline $\mathbf{P 2} \times \mathbf{P 6}$ & $10.73 *$ & -1.37 & 4.65 & 2.62 & -3.14 & -0.19 \\
\hline $\mathbf{P} 2 \times \mathbf{P 7}$ & -0.45 & -2.99 & -1.72 & -7.74 & -4.72 & $-6.26^{*}$ \\
\hline $\mathbf{P} 2 \times \mathbf{P 8}$ & $22.02 * *$ & 4.60 & $13.27 * *$ & $13.10^{* *}$ & 2.73 & $8.04 * *$ \\
\hline $\mathbf{P} 2 \times \mathbf{P 9}$ & $12.47 * *$ & $-9.67 * *$ & 1.35 & 4.24 & $-11.29 * *$ & -3.33 \\
\hline $\mathbf{P} 2 \times \mathbf{P} 10$ & 2.68 & 2.84 & 2.76 & -4.83 & 1.00 & -1.99 \\
\hline P3 $\times$ P4 & $12.38^{* *}$ & $-12.39 * *$ & -0.06 & 4.16 & $-13.95^{* *}$ & -4.68 \\
\hline P3 $\times$ P5 & 2.68 & $9.88 * *$ & $6.30^{*}$ & -4.83 & 7.92 & 1.39 \\
\hline $\mathbf{P 3} \times \mathbf{P 6}$ & $-8.53 *$ & $9.04 * *$ & 0.30 & $-15.22 * *$ & $7.09 *$ & -4.33 \\
\hline $\mathbf{P 3} \times \mathbf{P 7}$ & $-8.53 *$ & $-16.75 * *$ & $-12.66^{* *}$ & $-15.22 * *$ & $-18.24 * *$ & $-16.69 * *$ \\
\hline P3 $\times$ P8 & $10.73 *$ & -2.69 & 3.99 & 2.62 & -4.43 & -0.82 \\
\hline $\mathbf{P 3} \times \mathbf{P 9}$ & $-15.79 * *$ & $-17.16^{* *}$ & $-16.48 * *$ & $-21.95 * *$ & $-18.65^{* *}$ & $-20.34 * *$ \\
\hline $\mathbf{P 3} \times \mathbf{P 1 0}$ & 2.68 & -6.27 & -1.81 & -4.83 & $-7.94^{*}$ & $-6.35^{*}$ \\
\hline P4 × P5 & 6.99 & $6.90 *$ & $6.94 * *$ & -0.84 & 4.98 & 2.00 \\
\hline P4 $\times$ P6 & 2.68 & 1.73 & 2.20 & -4.83 & -0.09 & -2.52 \\
\hline P4 $\times$ P7 & $20.25^{* *}$ & $18.00 * *$ & $19.12^{* *}$ & $11.45^{* *}$ & $15.89 * *$ & $13.62 * *$ \\
\hline P4 × P8 & $-18.80 * *$ & $8.30^{*}$ & $-5.19^{*}$ & $-24.74 * *$ & $6.36^{*}$ & $-9.57 * *$ \\
\hline P4 × P9 & -1.66 & $6.90^{*}$ & 2.64 & $-8.85^{*}$ & 4.98 & -2.10 \\
\hline $\mathbf{P 4} \times \mathbf{P 1 0}$ & -5.09 & -1.67 & -3.37 & $-12.04 * *$ & -3.43 & $-7.84 * *$ \\
\hline $\mathrm{P} 5 \times \mathrm{P6}$ & $16.45^{* *}$ & -5.07 & $5.64 *$ & $7.93 *$ & $-6.77 *$ & 0.76 \\
\hline $\mathbf{P 5} \times \mathbf{P 7}$ & $-8.74 *$ & 2.33 & -3.18 & $-15.41 * *$ & 0.50 & $-7.65^{* *}$ \\
\hline $\mathrm{P} 5 \times \mathrm{P} 8$ & $-15.67 * *$ & $9.70 * *$ & -2.92 & $-21.84 * *$ & $7.74 *$ & $-7.41 * *$ \\
\hline P5 $\times$ P9 & $16.24 * *$ & $13.82 * *$ & $15.02 * *$ & 7.74 & $11.79 * *$ & $9.71 * *$ \\
\hline $\mathrm{P} 5 \times \mathrm{P} 10$ & -5.09 & $-12.45^{* *}$ & $-8.79 * *$ & $-12.04 * *$ & $-14.01 * *$ & $-13.00^{* *}$ \\
\hline $\mathbf{P 6} \times \mathbf{P 7}$ & $-10.73^{*}$ & -1.07 & $-5.88^{*}$ & $-17.26^{* *}$ & -2.84 & $-10.23^{* *}$ \\
\hline $\mathrm{P6} \times \mathrm{P8}$ & $-8.53 *$ & -0.93 & -4.71 & $-15.22 * *$ & -2.70 & $-9.11^{* *}$ \\
\hline $\mathrm{P6} \times \mathrm{P9}$ & -0.45 & $-9.37 * *$ & -4.93 & -7.74 & $-10.99 * *$ & $-9.32 * *$ \\
\hline $\mathrm{P} 6 \times \mathrm{P} 10$ & -1.36 & -1.97 & -1.66 & $-8.57 *$ & -3.72 & $-6.21 *$ \\
\hline $\mathbf{P 7} \times \mathbf{P 8}$ & -3.89 & $-18.06 * *$ & $-11.01 * *$ & $-10.92 * *$ & $-19.53 * *$ & $-15.12^{* *}$ \\
\hline $\mathbf{P 7} \times \mathbf{P 9}$ & 6.69 & $-7.46^{*}$ & -0.42 & -1.12 & $-9.12 * *$ & $-5.02 *$ \\
\hline P7 $\times$ P10 & $-16.87 * *$ & -1.67 & $-9.24 * *$ & $-22.95 * *$ & -3.43 & $-13.43 * *$ \\
\hline $\mathrm{P8} \times \mathrm{P9}$ & -8.23 & $-12.36 * *$ & $-10.30 * *$ & $-14.94 * *$ & $-13.93 * *$ & $-14.45^{* *}$ \\
\hline $\mathrm{P} 8 \times \mathrm{P} 10$ & $16.45^{* *}$ & $14.42 * *$ & $15.43 * *$ & $7.93 *$ & $12.37 * *$ & $10.10 * *$ \\
\hline $\mathrm{P} 9 \times \mathrm{P} 10$ & -8.23 & $10.60 * *$ & 1.23 & $-14.94 * *$ & $8.62 * *$ & -3.45 \\
\hline
\end{tabular}

D1 , D2 and com. refer to first , second planting dates and combined data, respectively.

* and ** significant at 0.05 and 0.01 levels of probability, respectively. 
Table 9. Heterosis for oil (\%) relative to S.C. 10 and S.C. $30 \mathrm{K8}$ in two planting dates as well as combined data

\begin{tabular}{|c|c|c|c|c|c|c|}
\hline \multirow{3}{*}{$\begin{array}{l}\text { Trait } \\
\text { Cross }\end{array}$} & \multicolumn{6}{|c|}{ Oil (\%) } \\
\hline & \multicolumn{3}{|c|}{ Heterosis (\%) relative to S.C. 10} & \multicolumn{3}{|c|}{ Heterosis (\%) relative to S.C. 30 K 8} \\
\hline & D1 & D2 & Com. & D1 & D2 & Com. \\
\hline$\overline{\mathbf{P 1} \times \mathbf{P 2}}$ & $23.17^{*}$ & 17.10 & $20.02 * *$ & 18.43 & 15.61 & $16.99 *$ \\
\hline $\mathbf{P 1} \times \mathbf{P 3}$ & 0.15 & 14.45 & 7.56 & -3.70 & 12.99 & 4.84 \\
\hline $\mathbf{P 1} \times \mathbf{P 4}$ & -8.85 & $30.11 * *$ & 11.35 & -12.36 & $28.46^{* *}$ & 8.53 \\
\hline $\mathbf{P 1} \times \mathbf{P 5}$ & -2.00 & 12.88 & 5.71 & -5.77 & 11.44 & 3.04 \\
\hline $\mathbf{P 1} \times \mathbf{P 6}$ & 3.08 & 6.22 & 4.71 & -0.89 & 4.87 & 2.06 \\
\hline $\mathbf{P 1} \times \mathbf{P 7}$ & 3.93 & -12.80 & -4.75 & -0.07 & -13.91 & -7.16 \\
\hline $\mathbf{P 1} \times \mathbf{P 8}$ & 7.54 & -1.29 & 2.97 & 3.40 & -2.54 & 0.36 \\
\hline $\mathbf{P 1} \times \mathbf{P 9}$ & 4.31 & -3.93 & 0.04 & 0.30 & -5.16 & -2.49 \\
\hline $\mathbf{P} 1 \times \mathbf{P 1 0}$ & -4.93 & -0.93 & -2.86 & -8.59 & -2.19 & -5.31 \\
\hline $\mathbf{P 2} \times \mathbf{P 3}$ & -19.78 & 9.94 & -4.38 & $-22.87 *$ & 8.55 & -6.79 \\
\hline P2 $\times$ P4 & -14.40 & 7.94 & -2.82 & -17.69 & 6.57 & -5.28 \\
\hline $\mathbf{P 2} \times \mathbf{P 5}$ & -7.54 & 14.09 & 3.67 & -11.10 & 12.64 & 1.05 \\
\hline $\mathbf{P 2} \times \mathbf{P 6}$ & 14.01 & 13.81 & 13.90 & 9.62 & 12.36 & 11.02 \\
\hline $\mathbf{P} 2 \times \mathbf{P 7}$ & 0.69 & $22.17^{*}$ & 11.83 & -3.18 & $20.62 *$ & 9.00 \\
\hline $\mathbf{P 2} \times \mathbf{P 8}$ & -1.39 & 4.08 & 1.45 & -5.18 & 2.75 & -1.12 \\
\hline $\mathbf{P} 2 \times \mathbf{P 9}$ & $-42.11 * *$ & 3.08 & $-18.69 *$ & $-44.34 * *$ & 1.77 & $-20.74 * *$ \\
\hline $\mathbf{P} 2 \times \mathbf{P} 10$ & -14.01 & -6.37 & -10.05 & -17.32 & -7.56 & -12.32 \\
\hline P3 $\times$ P4 & -6.08 & -5.08 & -5.56 & -9.70 & -6.29 & -7.95 \\
\hline P3 $\times$ P5 & 8.62 & 17.81 & 13.39 & 4.44 & 16.31 & 10.52 \\
\hline $\mathbf{P 3} \times \mathbf{P 6}$ & 14.47 & -4.94 & 4.41 & 10.07 & -6.14 & 1.77 \\
\hline P3 $\times \mathbf{P 7}$ & -9.39 & 16.81 & 4.19 & -12.88 & 15.32 & 1.55 \\
\hline $\mathbf{P 3} \times \mathbf{P 8}$ & 0.85 & 16.02 & 8.71 & -3.03 & 14.55 & 5.96 \\
\hline $\mathbf{P 3} \times \mathbf{P 9}$ & 5.31 & 15.24 & 10.46 & 1.26 & 13.77 & 7.66 \\
\hline $\mathbf{P 3} \times \mathbf{P} 10$ & 1.62 & 16.02 & 9.08 & -2.29 & 14.55 & 6.32 \\
\hline P4 × P5 & 0.08 & 9.59 & 5.01 & -3.77 & 8.19 & 2.35 \\
\hline $\mathbf{P 4} \times \mathbf{P 6}$ & 18.09 & 1.29 & 9.38 & 13.55 & 0.00 & 6.61 \\
\hline $\mathbf{P 4} \times \mathbf{P 7}$ & 17.17 & 7.01 & 11.90 & 12.66 & 5.65 & 9.07 \\
\hline P4 $\times$ P8 & 3.16 & 13.38 & 8.45 & -0.81 & 11.94 & 5.71 \\
\hline P4 $\times$ P9 & -17.01 & $18.88^{*}$ & 1.59 & -20.21 & 17.37 & -0.98 \\
\hline $\mathbf{P} 4 \times \mathbf{P} 10$ & 19.09 & 9.30 & 14.02 & 14.51 & 7.91 & 11.13 \\
\hline P5 $\times$ P6 & -11.24 & 2.07 & -4.34 & -14.66 & 0.78 & -6.76 \\
\hline $\mathbf{P 5} \times \mathbf{P 7}$ & $28.25^{*}$ & -5.72 & 10.64 & $23.32 *$ & -6.92 & 7.84 \\
\hline $\mathrm{P} 5 \times \mathrm{P} 8$ & 19.86 & 7.15 & 13.27 & 15.25 & 5.79 & 10.41 \\
\hline P5 $\times$ P9 & 3.39 & $22.10^{*}$ & 13.09 & -0.59 & $20.55^{*}$ & 10.23 \\
\hline $\mathrm{P} 5 \times \mathrm{P} 10$ & 0.23 & $21.10^{*}$ & 11.05 & -3.63 & $19.56^{*}$ & 8.24 \\
\hline P6 $\times$ P7 & $-22.63 *$ & -6.29 & -14.16 & $-25.61 *$ & -7.49 & $-16.34 *$ \\
\hline $\mathrm{P} 6 \times \mathbf{P 8}$ & 9.08 & 9.59 & 9.34 & 4.89 & 8.19 & 6.58 \\
\hline P6 $\times$ P9 & 21.71 & -2.43 & 9.20 & 17.02 & -3.67 & 6.43 \\
\hline $\mathrm{P} 6 \times \mathrm{P} 10$ & 4.08 & 4.94 & 4.52 & 0.07 & 3.60 & 1.88 \\
\hline $\mathbf{P 7} \times \mathbf{P 8}$ & -5.31 & 12.66 & 4.00 & -8.96 & 11.23 & 1.37 \\
\hline $\mathbf{P 7} \times \mathbf{P 9}$ & 2.69 & $-32.55^{* *}$ & $-15.57 *$ & -1.26 & $-33.40^{* *}$ & $-17.71^{*}$ \\
\hline $\mathbf{P} 7 \times \mathbf{P 1 0}$ & 21.48 & $-19.96^{*}$ & 0.00 & 16.80 & $-20.97 *$ & -2.53 \\
\hline $\mathrm{P8} \times \mathrm{P9}$ & 0.08 & -11.44 & -5.90 & -3.77 & -12.57 & -8.28 \\
\hline $\mathrm{P} 8 \times \mathrm{P} 10$ & $25.02 *$ & -16.81 & 3.34 & 20.21 & -17.87 & 0.72 \\
\hline $\mathrm{P} 9 \times \mathrm{P} 10$ & 10.32 & -3.43 & 3.19 & 6.07 & -4.66 & 0.58 \\
\hline
\end{tabular}

D1 , D2 and com., refer to first , second planting dates and combined data, respectively.

* and ** significant at 0.05 and 0.01 levels of probability, respectively. 
Table 10. Correlation matrix between grain yield/plant and other important agronomic characters in maize hybrids combined over two planting dates

\begin{tabular}{|c|c|c|c|c|c|c|c|c|c|c|c|c|}
\hline Trait & 1 & 2 & 3 & 4 & 5 & 6 & 7 & 8 & 9 & 10 & 11 & 12 \\
\hline 1- Grain yield/ plant & 1.0000 & & & & & & & & & & & \\
\hline 2- Days to $50 \%$ tasseling & -0.0415 & 1.0000 & & & & & & & & & & \\
\hline 3- Days to $50 \%$ silking & -0.0390 & $0.9406 *$ & $* 1.0000$ & & & & & & & & & \\
\hline 4- Plant height & 0.0176 & -0.1353 & -0.1416 & 1.0000 & & & & & & & & \\
\hline 5- Ear height & 0.1771 & -0.1228 & -0.1030 & $0.8512 *$ & $* 1.0000$ & & & & & & & \\
\hline 6- Chlorophyll content & $0.6372 *$ & $*-0.1731$ & -0.1722 & 0.2993 & $0.4855 *$ & $* 1.0000$ & & & & & & \\
\hline 7- Ear length & $0.5116 *$ & $* 0.0181$ & 0.0806 & 0.0717 & 0.0200 & 0.2054 & 1.0000 & & & & & \\
\hline 8- Ear diameter & $0.6074 *$ & $* 0.0659$ & 0.0424 & -0.1738 & -0.2066 & 0.2580 & 0.1580 & 1.0000 & & & & \\
\hline 9- No. of rows/ ear & $0.4794 *$ & $*-0.1840$ & -0.1757 & 0.0274 & 0.0554 & $0.5134 *$ & $* 0.3638 *$ & 0.2633 & 1.0000 & & & \\
\hline 10- No. of grains/ row & $0.6909 *$ & $*-0.1511$ & -0.1164 & 0.0761 & 0.1516 & $0.4235 *$ & $* 0.7720 *$ & $* 0.3531 *$ & $* 0.4148 *$ & $* 1.0000$ & & \\
\hline 11- 100 grain weight & $0.7344 *$ & * 0.0793 & 0.0428 & -0.0973 & 0.0422 & $0.4435 *$ & $* 0.0465$ & $0.5978 *$ & $* 0.0713$ & 0.1650 & 1.0000 & \\
\hline 12- Shelling (\%) & $0.4021 *$ & * 0.1920 & 0.2009 & 0.0377 & 0.2266 & $0.3575 *$ & 0.2328 & 0.1867 & 0.1832 & $0.3851 *$ & $* * 0.2612$ & 1.0000 \\
\hline
\end{tabular}

Significant and positive correlation values were also detected for ear length and each of number of rows/ear $\left(0.3638^{*}\right)$ and number of grains/row $\left(0.7720^{* *}\right)$. The association between ear diameter and each of number of grains/row $\left(0.3531^{*}\right)$ and 100 grain weight $\left(0.5978^{* *}\right)$ was positive and significant. Furthermore, positive and significant correlation coefficient was found between number of grains/ row and each of number of rows/ear $\left(0.4148^{* *}\right)$ and shelling (\%) $\left(0.3851^{* *}\right)$. In this connection, significantly positive correlation coefficients between grain yield and its contributing traits were previously reported by several investigators, among those Beshay (2010), El-Badawy and Mehasen (2011), Filipovic et al. (2014) and Sayedzavar et al. (2015).

Many statistical methods are used to study the relation between independent and dependent variables. Factor analysis is different; it is used to study the patterns of relationship among many dependent variables, with the goal of discovering something about the nature of the independent variables that affect them, even though those independent variables were not measured directly. The inferred independent variables are called factors. Thus, factor analysis is a statistical method used because of its power to elicit underlying multivariate structures (Walton, 1972).

The factor analysis technique divided the studied variables into four main factors (Tables 11, 12 and Fig. 1). These four factors accounted for $77.83 \%$ of the total variability in the dependence structure of maize grain yield. The first factor included four variables and accounted for $21.15 \%$. These variables were ear length $(32.15 \%)$, number of rows ear $(22.09 \%)$, number of grains row $^{-1}(32.01 \%)$ and shelling (\%) $(13.75 \%)$. It is clear that these variables had a high loading coefficients and participate much more on the dependence structure. Most of these variables exhibited positive and significant correlation values with maize grain yield as previously mentioned .

The second factor consists of two variables and accounted for $19.73 \%$ of the total variability of maize grain yield. These two variables were plant height (48.02\%), and ear height (51.98\%) as shown in Table 12.

The third factor included two variables and accounted for $19.00 \%$ of total variance. These variables were days to $50 \%$ tasseling $(49.79 \%)$ and days to $50 \%$ silking (50.21\%).

Three variables were loaded in the fourth factor and accounted for $17.95 \%$ of the total variance of the dependence structure. These variables were chlorophyll content $(25.36 \%)$, ear diameter $(34.54 \%)$ and 100 grain weight (40.11\%).

It could be concluded that selection for the most important yield traits particularly number of rows/ear, number of grains/row, 100 grain weight and shelling (\%) would lead to maximizing total maize grain yield. These results agree with those obtained by Beshay (2010) El-Badawy and Mehasen (2011), Beiragi et al. (2012), Khodarahmpour (2013), Filipovic et al. (2014) and Sayedzavar et al. (2015). 
Table 11. Principal factor matrix after orthogonal rotations for studied characters of maize

\begin{tabular}{lrrrrr}
\hline Variable & \multicolumn{4}{c}{ Common factors coefficient } & \multirow{2}{*}{ Communality (\%) } \\
\cline { 2 - 5 } & Factor 1 & Factor 2 & Factor 3 & Factor 4 & \\
\hline Days to 50\% tasseling & -0.085 & -0.085 & 0.957 & 0.040 & 0.933 \\
Days to 50\% silking & -0.032 & 0.081 & 0.965 & -0.003 & 0.939 \\
Plant height (cm) & 0.023 & 0.896 & -0.076 & -0.139 & 0.829 \\
Ear height (cm) & 0.039 & 0.970 & -0.032 & 0.011 & 0.944 \\
Chlorophyll content & 0.362 & 0.518 & -0.170 & 0.569 & 0.752 \\
Ear length (cm) & 0.898 & -0.017 & 0.119 & -0.080 & 0.828 \\
Ear diameter (cm) & 0.224 & -0.026 & 0.021 & 0.775 & 0.718 \\
No. of rows/ ear & 0.617 & 0.046 & -0.243 & 0.234 & 0.497 \\
No. of grains/ row & 0.897 & 0.081 & -0.059 & 0.176 & 0.840 \\
100 grain weight (g) & -0.021 & 0.021 & 0.064 & 0.900 & 0.815 \\
Shelling (\%) & 0.384 & 0.262 & 0.354 & 0.356 & 0.468 \\
Variance ratio & 21.15 & 19.73 & 19.00 & 17.95 & 77.83 \\
\hline
\end{tabular}

Table 12. Summary of factor loading for some important traits of maize

\begin{tabular}{|c|c|c|c|}
\hline$\overline{\text { Variable }}$ & Loading & $\begin{array}{c}\text { Percentage of total } \\
\text { communiality }\end{array}$ & \\
\hline Factor 1 & & & 21.15 \\
\hline Ear length $(\mathrm{cm})$ & 0.898 & 32.15 & \\
\hline No. of rows/ ear & 0.617 & 22.09 & \\
\hline No. of grains/ row & 0.894 & 32.01 & \\
\hline Shelling (\%) & 0.384 & 13.75 & \\
\hline Factor 2 & & & 19.73 \\
\hline Plant height (cm) & 0.896 & 48.02 & \\
\hline Ear height (cm) & 0.970 & 51.98 & \\
\hline Factor 3 & & & 19.00 \\
\hline Days to $50 \%$ tasseling & 0.957 & 49.79 & \\
\hline Days to $50 \%$ silking & 0.965 & 50.21 & \\
\hline Factor 4 & & & 17.95 \\
\hline Chlorophyll content & 0.569 & 25.36 & \\
\hline Ear diameter (cm) & 0.775 & 34.54 & \\
\hline 100 grain weight (g) & 0.900 & 40.11 & \\
\hline Cummulative variance & & & 77.83 \\
\hline
\end{tabular}




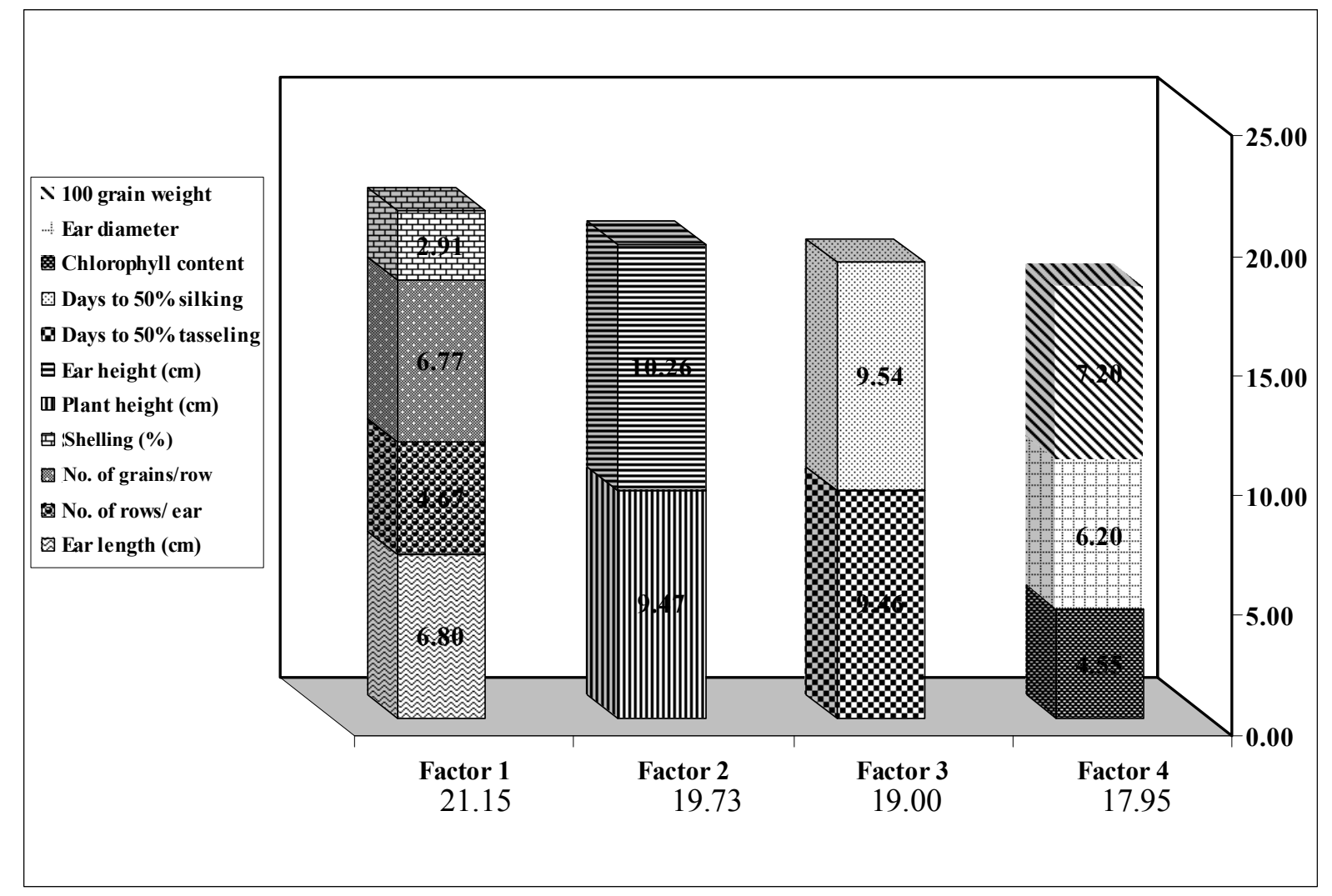

Fig. 1. Factor loading for some important characters of maize

\section{REFERENCES}

AOAC (1990), Official Methods of Analysis. $13^{\text {th }}$ Ed. Washington DC. Ass. Anal. Chem., USA.

Abd-Elaziz, M.A. (2014). Diallel analysis for some agronomic attribute of maize under different planting dates. Ph. D. Thesis, Fac. Agric., Benha Univ., Egypt.

Abdel-Moneam, M.A., M.S. Sultan, S.E. Sadek and M.S. Shalof (2014). Estimation of heterosis and genetic parameters for some flowering and vegetative traits in maize using the diallel cross method. J. Adv. in Nat. Sci., 2 (2): 157- 166.

Abrha, S.W. (2014). Standard heterosis of maize (Zea mays L.) inbred lines for grain yield and yield related traits in central rift valley of Ethiopia. J. Biol., Agric. and Healthcare, 4 (33): 31- 37.
Al-Falahy, M.A.H. (2015). Estimation combining ability, heterosis and some genetic parameters across four environments using full diallel cross method. Int. J. Pure Appl. Sci. Technol., 26 (1): 34- 44.

Ali, A., H. Rahman, L. Shah, K.A. Shah and S. Rehman (2014). Heterosis for grain yield and its attributing components in maize variety Azam using line $\mathrm{x}$ tester analysis method. Academic J. Agri. Res., 2 (11): 225- 230.

Beiragi, M.A., B.A. Sar, H.S. Geive, M.N. Alhossini, A. Rahmani and A.B. Gharibdoosti (2012). Application of the multivariate analysis method for some traits in maize. African J. Agric. Res., 7 (10): 1524- 1533.

Bekele, A. and T.N. Rao (2013). Heterosis study for grain, protein and oil improvement in selected genotypes of maize (Zea mays L.). J. Plant Sci., 1(4): 57- 63.

Beshay, M.Y. (2010). Response of some maize genotypes to nitrogen fertilization. $\mathrm{Ph}$. D Thesis, Fac. Agric. Benha Univ., Egypt. 
Cattell, E.B. (1965). Factor analysis: An introduction to essentials. I. The purpose and the underlying models. Biom., 21: 190- 215.

El-Badawy, M.E.M. (2013). Heterosis and combining ability in maize using diallel crosses among seven new inbred lines. Asian J. Crop Sci., 5(1): 1-13.

El-Badawy, M.E.M. and S.A.S. Mehasen (2011). Multivariate analysis for yield and its components in maize under zinc and nitrogen fertilization levels. Aust. J. Basic App. Sci., 5 (2): 3008- 3015.

Filipovic, M., M. Babic, N. Delic, G. Bekavac and V. Babic (2014). Determination relevant breeding criteria by the path and factor analysis in maize. Genetika, 46 (1): 49- 58.

Gomez, K.N. and A.A. Gomez (1984). Statistical Procedures for Agricultural Research. John. Wiley and Sons Inc., New York, $2^{\text {nd }}$ Ed.

Khodarahmpour, Z. (2013). Study of some quantitative traits in maize (Zea mays L.) inbred lines under the drought stress using multivariate analysis. Int. J. Agric. Crop Sci., 5 (14): 1547- 1552.

Lahane, G.R., R.M. Chauhan and J.M. Patel (2014). Combining ability and heterosis studies for yield and quality traits in quality protein maize. J. Agric. Res., 1(3): 135- 138.

Rajesh, V., S.S. Kumar, V.N. Reddy and A. SivaSankar (2014). Heterosis studies for grain yield and its component traits in single cross hybrids of maize (Zea mays L.). Int. J. Plant, Animal, Env. Sci., 4 (1): 304- 306.

Ram, L., R. Singh, S.K. Singh and R.P. Srivastava (2015). Heterosis and combining ability studies for quality protein in maize. J. Crop Breeding and Genet., 1 (2): 8- 25.

Reddy, V.R., F. Jabeen and M.R. Sudarshan (2015). Heterosis studies in diallel crosses of maize for yield and yield attributing traits in maize (Zea mays L.) over locations. Int. J. Agric. Env. Biotech., 8(2): 271- 283.

Sayedzavar, J., M. Norouzi and S. Aharizad (2015). Relationships of morphological characters and yield components in corn hybrids under water deficit stress. Biological Forum- An Int. J., 7 (1): 1512- 1519.

Sedhom, S.A., M.H. Tag El-Din, M.E. El Badawy and M.A. El Bakey (2012). Breeding for grain yield, yield components and quality traits in yellow maize (Zea mays L.). Proc. $13^{\text {th }}$ Int. Conf. Agron. Fac. Agric. Benha Univ., Egypt, 332- 351.

Snedecor, G.W. and W.G. Cochran (1967). Statistical Methods. $6^{\text {th }}$ Ed., Iowa State Univ., Press. Ames, Iowa, USA.

Ulaganathan, V. and S.M. Ibrahim (2014). Heterosis studies for physiological and quality parameters in quality protein of maize hybrids (Zea mays L.). Biosci., 7 (19): 29362940.

Walton, P.D. (1972). Factor analysis of yield in spring wheat (Triticum aestivum L.). Crop Sci., 12: 731- 733.

Zaid, I.U., H. Rahman, S. Khan, S.U. Khan, G. Ullah, M. Rehman, R. Ullah and N. Ahmed (2014). Heterosis response of three-way maize hybrids for grain yield and yield components. J. Agric. Sci. Appl., 3 (1): 2429. 


\section{قــوة الهجيـن والتحليـل العاملى لبعض الصفات الهامة فى هجــن جـــــــة مسن الـــرة الثــامية}

\section{يوستينا سبدهم أسعد سيدهم - محمد محمد عبدالحميد علي - حسن عوده عواد ـ حسن أحمد ربيع}

$$
\text { قسم المحاصيلـ كلية الزر اعة - جامعة الزقازيق - مصر }
$$

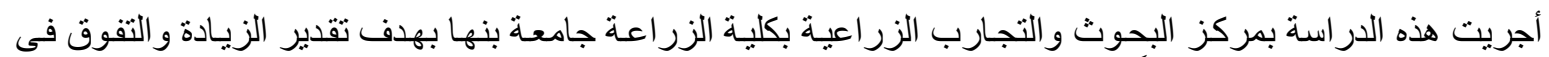
P

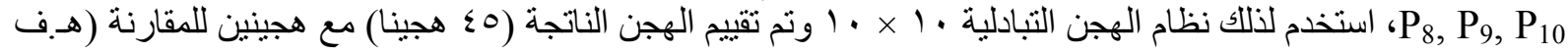

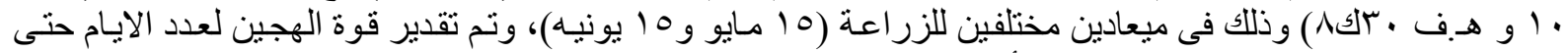

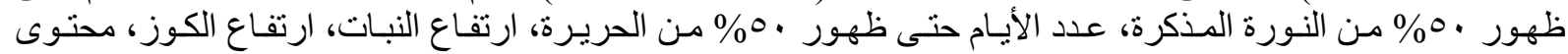

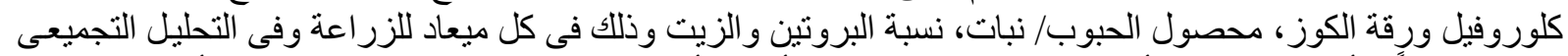

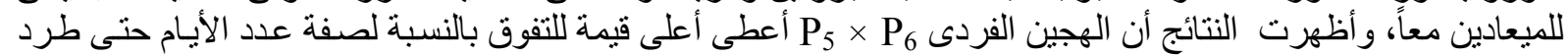

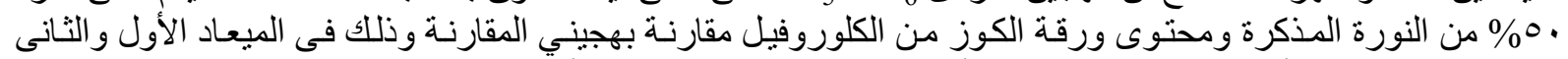

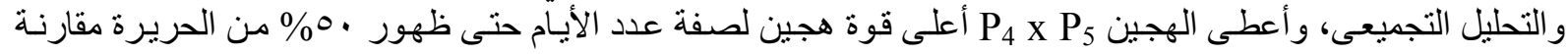

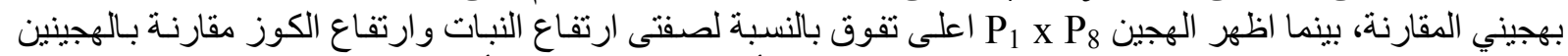

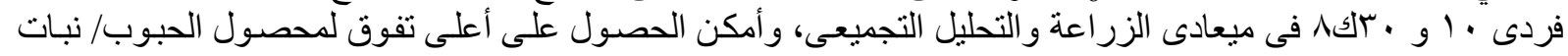

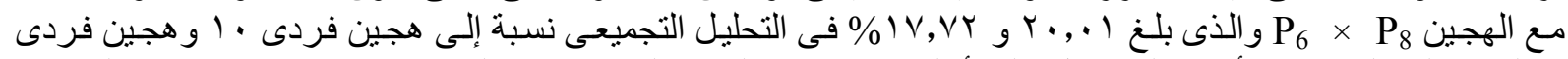

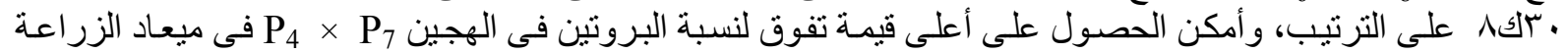

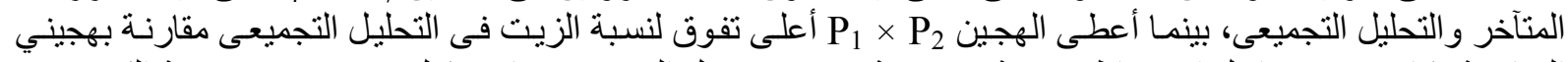

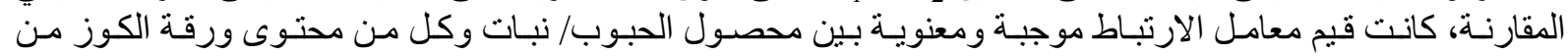

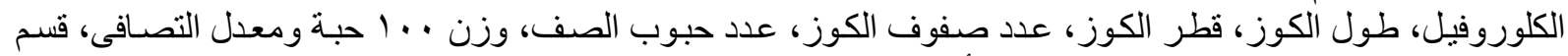

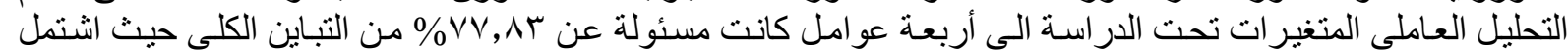

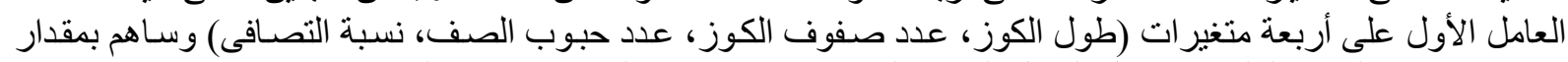

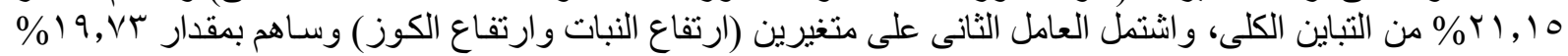

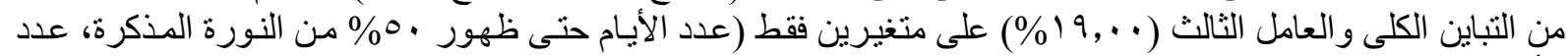

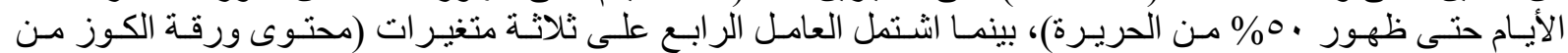

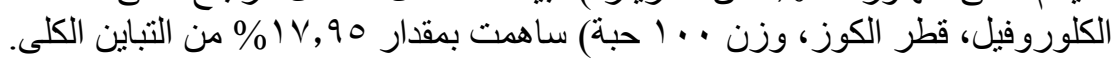

أستاذ تربية المحاصيل - كلية الزر اعة بمشتهر - جامعة بنها. أستاذ تربية المحاصيل المتفرغ غية - كلية الزر اعة اعة - جامعة الزقازيق. 